\title{
Modelagem Matemática para otimização de desempenho de móbiles em descida de rampa
}

\section{Mathematical modelling to optimize mobile performance on downhill ramps}

\author{
Maurício Garcia Chiarello \\ Universidade de Franca (UNIFRAN), Departamento de Engenharia, Franca, SP, Brasil \\ (D) https://orcid.org/0000-0002-2982-5853, chiarello@scientiaestudia.org.br
}

\section{Informações do Artigo \\ Como citar este artigo \\ CHIARELLO, Maurício. Modelagem \\ Matemática para otimização de desempenho \\ de móbiles em descida de rampa. REMAT: \\ Revista Eletrônica da Matemática, Bento \\ Gonçalves, RS, v. 7, n. 2, p. e3002, 4 ago. \\ 2021. DOI: \\ https://doi.org/10.35819/remat2021v7i2id4800}

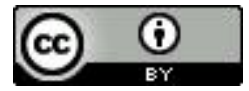

Histórico do Artigo

Submissão: 16 de dezembro de 2020

Aceite: 17 de maio de 2021.

\section{Palavras-chave}

Móbiles em Rampa

Competição em Descida Livre

Equacionamento Dinâmico

Influência da Massa

Otimização de Desempenho

\section{Resumo}

O presente estudo analisa móbiles, dotados ou não de força de tração, que descem uma rampa inclinada sob a ação da gravidade, sujeitos às forças de arrasto e atrito de rolamento. $A$ partir do equacionamento dinâmico e da obtenção das equações de velocidade e posição, investigou-se o efeito que possui a massa dos móbiles sobre seu desempenho em uma eventual competição, entendendo por desempenho a distância percorrida pelos móbiles em um intervalo fixo de tempo, previamente estipulado. Foi possível concluir que, quando os móbiles não são motorizados, o desempenho cresce continuamente com a massa. Por esse motivo, é necessário padronizar o valor de massa dos veículos competidores para garantia de isonomia. Já quando são dotados de força de tração constante, pode haver um valor de massa capaz de maximizar o desempenho, sendo por isso interessante deixar para as equipes competidoras a tarefa de determinar o valor de massa mais vantajoso para seus veículos. Foram avaliadas as condições sob as quais o desempenho admite ser otimizado e uma expressão analítica para determinação da massa dos móbiles que maximiza o desempenho foi obtida.

\section{Keywords}

Ramp Mobiles

Gravity Car Race

Dynamic Equation

Mass Influence

Performance Optimization

\begin{abstract}
The present study analyses mobiles, with or without traction force, that descend an inclined ramp under the action of gravity, subject to drag forces and kinetic friction. From the dynamic equation and the obtainment of the speed and position equations, the effect of the mobile mass on their performance in an eventual competition was investigated, understanding by performance the distance travelled by the mobiles in a fixed interval of time, previously stipulated. It was possible to conclude that, when the mobiles are not motorized, the performance grows continuously with the mass. For this reason, it is necessary to standardize the mass value of competing vehicles to guarantee isonomy. When vehicles are equipped with constant traction force, there may be a mass value capable of maximizing performance. Therefore, it is interesting to leave it up to competing teams to determine the most advantageous mass value for their vehicles. The conditions under which the performance is supposed to be optimized were evaluated and an analytical expression for determining the mass that maximizes the performance was obtained.
\end{abstract}




\section{Introdução}

La sfida consistere in partecipare a una competizione singolare, in cui il vincitore è colui che, giocando, dissipa al meglio la voragine del tempo.

As metodologias ativas de aprendizado baseadas em projetos interdisciplinares têm sido adotadas pelos cursos de ensino superior do país, nos últimos anos, com grande proveito para a formação na área de ciências exatas, fornecendo bons resultados. Nos cursos de engenharia, em particular, competições vêm sendo propostas, já há alguns anos, visando estimular os alunos a se dedicarem com grande empenho nos projetos interdisciplinares. Entre tantas categorias, podem ser mencionadas as competições de veículos de baixo consumo, cujo desafio consiste em otimizar a eficiência energética, buscando alcançar as melhores marcas de economia, seja de combustível, seja de energia elétrica. Também são muito comuns as competições envolvendo veículos baja, nas quais diversos quesitos do veículo projetado e executado pelas equipes participantes são avaliados, tais como segurança, conforto, aceleração, tração, suspensão e resistência estrutural.

O trabalho que aqui se apresenta foi elaborado tendo em vista uma modalidade particular dessas competições, conhecidas por gravity car race, nas quais veículos não motorizados descem uma rampa pela ação exclusiva da gravidade. Ao realizar um estudo analítico do movimento de móbiles em descida de rampa, ele foi concebido com o propósito de oferecer os recursos da análise matemática para um projeto interdisciplinar mais amplo, capaz de integrar diversas disciplinas de um curso de engenharia. O projeto seria desenvolvido visando a disputa de uma competição pautada nos seguintes termos: considerando que se possa dispor de uma rampa inclinada relativamente longa e com inclinação praticamente constante, os alunos do curso, formando equipes, seriam convidados a projetar e a fabricar móbiles sobre rodas, pilotados ou teleguiados, para participar de uma competição na qual o vencedor seria o móbile da equipe capaz de, em um intervalo fixo de tempo previamente definido, percorrer a maior distância sobre a rampa. As mais diferentes competências técnicas e habilidades profissionais precisariam então ser mobilizadas, contribuindo para a boa formação profissional.

O estudo analítico realizado no presente trabalho considera, em um primeiro momento, que os móbiles sejam dotados de um propulsor capaz de fornecer uma força de tração constante - esta é uma modalidade de competição, vale notar, ainda não teorizada na literatura existente, até onde sabe o autor. Em um segundo momento, considera que os móbiles não sejam motorizados, e que, portanto, desçam a rampa pela ação exclusiva da gravidade, como normalmente acontece nos torneios de gravity car race. Em ambos os casos, tanto a força de atrito de rolamento como a força de arrasto aerodinâmico foram levadas em conta. O fulcro da análise empreendida consistiu em investigar o efeito que possui a massa dos veículos sobre o 
desempenho. Além dessa investigação, no entanto, também foi analisada a sensibilidade do desempenho à variação dos coeficientes de atrito e de arrasto, com o propósito se fornecer balizas teóricas para aperfeiçoamento dos veículos competidores.

$\mathrm{Na}$ bibliografia que aborda as competições de veículos em descida livre (gravity car race), há um bom número de publicações cujo teor dominante consiste em fornecer conselhos e orientações práticas para melhorar o desempenho dos veículos competidores. Esse é o caso de Pedigo (2002), Polycarpou e Solzak (2006), Meade (2006) e Torvi (2011), para mencionar apenas as publicações mais relevantes cujo teor dominante é o de aconselhamento prático. No entanto, raros são os trabalhos publicados até a presente data que equacionam o desempenho com rigor científico, de modo a fundamentar teoricamente a razão pela qual certas modificações podem promover ganho de desempenho efetivo. Entre esses poucos trabalhos, vale mencionar Coletta e Evans (2008), Martin (2015), Jobe (2004) e Cowley (1989). Pelo rigor no tratamento analítico, merece destaque o estudo de Mann, Gibbs e Sah (2012). Partindo do balanço energético, o equacionamento presente nesse estudo leva em conta até mesmo o momento de inércia das rodas e considera a força de arrasto como proporcional ao quadrado da velocidade instantânea. Consequentemente, a equação diferencial que modela a velocidade dos móbiles deixa de ser linear e a solução analítica, obtida para as equações de velocidade e posição, embora rigorosa, assume uma forma demasiadamente complexa. Essa complexidade da solução dificulta o tratamento analítico do problema. Há também alguns estudos que recorrem a métodos numéricos para resolução do equacionamento dinâmico. É o caso de Driscoll et al. (2016), que também considera o arrasto como proporcional ao quadrado da velocidade e adota o método de Euler para resolver a equação diferencial da velocidade.

Com o objetivo de determinar um conjunto de fórmulas matemáticas que, expressando o efeito que possui a variação dos parâmetros dos veículos sobre seu desempenho, possam ser convenientemente empregadas para otimização do desempenho, optou-se neste estudo por uma abordagem analítica do equacionamento dinâmico. Para contornar a complexidade resultante de um equacionamento dinâmico rigoroso, duas simplificações foram adotadas: o momento de inércia das rodas foi desprezado e a força de arrasto foi considerada diretamente proporcional à velocidade. Essa modelagem, que pode não se aplicar satisfatoriamente em um bom número de situações, tem a vantagem de permitir uma solução analítica relativamente simples, a partir da qual o efeito da variação dos parâmetros sobre o desempenho pode ser mais facilmente investigado. Embora os resultados encontrados possam não ser exatos, em virtude da modelagem simplificadora adotada, são capazes de fornecer balizas gerais de comportamento bastante úteis tanto para aprimoramento dos veículos, em busca de ganho de desempenho, como para definição dos critérios gerais de uma determinada competição.

A partir dos resultados obtidos pelo equacionamento analítico, sugestões puderam ser feitas para elaboração de critérios mais apropriados para cada uma das modalidades de 
competição estudadas. Nos roteiros propostos para definição desses critérios, buscou-se garantir que a competição seja a mais justa possível, evitando que características não controladas, ou insuficientemente controladas dos veículos venham a favorecer sub-repticiamente alguns competidores em detrimento de outros. Além disso, foram fornecidas estimativas da sensibilidade de desempenho às variações dos parâmetros dos veículos que podem ser úteis às equipes competidoras no trabalho de aprimoramento técnico visando aumento do desempenho.

\section{Fundamentação teórica}

Nas situações em que um móbile sobre rodas desce um plano inclinado sob a ação da gravidade, sem deslizamento ou escorregamento, deve-se considerar que seja atuante sobre ele uma força contrária ou resistente ao movimento, conhecida por força de atrito de rolamento. A resistência de rolamento corresponde à força necessária para vencer o atrito existente nos eixos, rolamentos e rodas. Ela depende da força normal de contato e é influenciada por inúmeros fatores, tais como a rugosidade da superfície do solo, o diâmetro e a largura das rodas, o material da banda de rodagem e sua deformação no contato com o solo (no que influencia a pressão dos pneus, se houver pneus), bem como o atrito nos eixos e rolamentos. É interessante notar que a força de atrito praticamente independe da velocidade do móbile. Pode-se reunir o efeito combinado desses diversos fatores em uma constante conhecida por coeficiente de atrito de rolamento. Desse modo, é possível escrever que a força de atrito de rolamento obedece à relação $F_{c}=\mu . N$, onde $\mu$ é o coeficiente de atrito de rolamento e $N$ a força normal.

Quando um móbile se desloca em um meio fluido, como é o caso do ar, é preciso ainda considerar que, além da força de atrito, atua sobre ele uma outra força de resistência ao movimento conhecida por força de arrasto. A força de arrasto $F_{a}$ é comumente modelada pela seguinte expressão, conhecida por equação do arrasto: $F_{a}=\frac{c_{d} A \rho}{2} v^{2}$. Nessa equação: $c_{d}$ é o coeficiente de arrasto do objeto, que depende fundamentalmente de sua forma geométrica; $A$ é a área de referência, que normalmente corresponde, para veículos, à área de projeção frontal; $\rho$ é a densidade de massa do fluido em que se move o objeto; $v$ é a velocidade do objeto em relação ao fluido.

Convém notar que a força de arrasto, diferentemente da força de atrito, depende da velocidade de deslocamento do objeto relativamente ao fluido, sendo modelada, normalmente, como proporcional ao quadrado da velocidade instantânea do objeto ${ }^{1}$. Neste breve estudo

\footnotetext{
1 Quase todas as obras de referência na área de mecânica dos fluidos tratam da força de arrasto e apresentam seu equacionamento. Em geral, o tópico é abordado no capítulo consagrado à fluidodinâmica. No livro de Brunetti (2008), a matéria é apresentada no capítulo 9 (p. 223). Nessa obra, o equacionamento da força de arrasto total, resultante da soma do arrasto de superfície com o arrasto de pressão, pode ser encontrada na secção 9.5 (p. 234).
} 
analítico, admite-se que a força de arrasto seja diretamente proporcional à velocidade instantânea do objeto. A equação que considera a força de arrasto como diretamente proporcional à velocidade, conhecida por arrasto de Stokes, mostra-se satisfatória apenas nas situações em que a velocidade do corpo é pequena relativamente à velocidade do fluido ou quando a viscosidade do fluido é muito alta. Ora, é bem possível que para alguns móbiles em descida de rampa a adoção do arrasto de Stokes não seja adequada. Em alguns casos, dependendo da velocidade máxima atingida pelo veículo e de seu perfil aerodinâmico, a aproximação poderá estar sujeita a severas limitações, o que precisará ser verificado experimentalmente por cada equipe participante. Ainda assim, optou-se aqui por adotar a equação do arrasto de Stokes em virtude da simplificação que ela promove no equacionamento dinâmico, permitindo a obtenção de uma solução analítica e de critérios bastante eficazes de análise. Como os coeficientes $c_{d}, A$ e $\rho$ devem manter-se invariáveis ao longo do deslocamento do objeto, a força de arrasto será, portanto, modelada pela relação $F_{a}=K v$, onde $K$ é a constante dada por $K=\frac{c_{d} A \rho}{2}$, aqui denominada constante de arrasto.

Admitindo-se, portanto, que a força de arrasto obedece à relação $F_{a}=K v$ e que a força de atrito de rolamento obedece à relação $F_{c}=\mu . N$, a soma das forças de resistência ao movimento $F_{r}$ na direção do deslocamento será dada por: $F_{r}=F_{a}+F_{c}=K v+\mu . N$. A imposição de que seja nula a resultante na direção perpendicular à direção do deslocamento permite escrever que $N=P \cdot \cos \theta=m g \cdot \cos \theta$, onde g é a aceleração da gravidade e $\theta$ a inclinação do plano em relação à horizontal. Desse modo, a força total de resistência ao deslocamento pode ser escrita como: $F_{r}=K v+\mu m g \cos \theta$. A Figura 1 representa de forma esquemática o conjunto das forças que aqui se considera atuantes sobre o móbile em movimento de descida de rampa.

Figura 1 - Forças atuantes sobre o móbile.

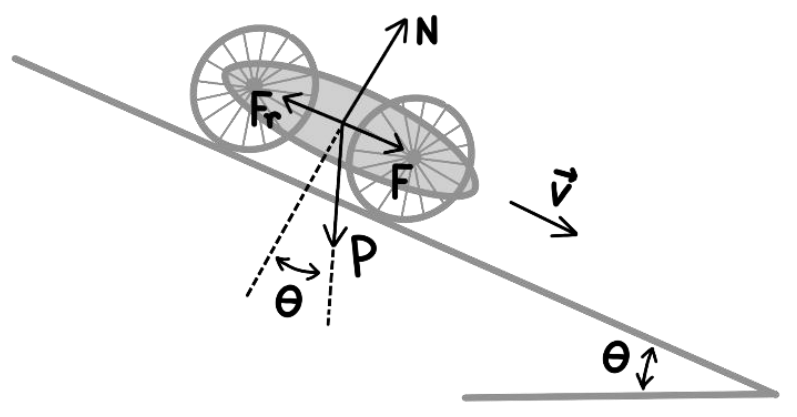

Fonte: Elaboração do autor (2021). 
Cumpre enfatizar que, no equacionamento dinâmico realizado no presente estudo, o momento de inércia das rodas não foi considerado. Assim sendo, os resultados obtidos serão tanto mais válidos quanto menor for o momento de inércia do conjunto das rodas do veículo. Presume-se, aqui, que as equipes competidoras deverão procurar minimizar o momento de inércia das rodas, reduzindo ao máximo sua massa total, posto que essa redução acarreta ganho de desempenho. Rodas de perfil delgado, com aro de liga leve, suportado por raios finos proporcionam baixo momento de inércia, sendo por isso recomendáveis.

\section{Análise de desempenho para móbiles dotados de força de propulsão}

Na presente secção, considerar-se-á que os móbiles em descida de rampa sejam dotados de um motor capaz de lhes fornecer uma força de propulsão $F$ constante. Com a finalidade de realizar um estudo analítico de desempenho, serão inicialmente determinadas as equações de velocidade e de posição.

\subsection{Equação de velocidade}

Duas são as forças promotoras de aceleração do movimento no sentido da descida de rampa: além da força de tração $F$, a componente da força peso na direção do deslocamento, dada por $P . \operatorname{sen} \theta=m g . \operatorname{sen} \theta$. A esse respeito, que se veja mais uma vez a Figura 1. Chamando $F_{p}$ a soma das forças propulsoras, então $F_{p}=F+m g . \operatorname{sen} \theta$. A força resultante $R$ sobre o móbile no sentido do deslocamento é dada pela diferença entre as forças propulsoras e as forças de resistência ao movimento. Assim sendo, $R=F_{p}-F_{r}=F+m g \cdot \operatorname{sen} \theta-(K v+\mu m g \cos \theta)$. A imposição da segunda lei de Newton, de acordo com a qual a força resultante R é igual ao produto da massa pela aceleração, isto é $R=m \frac{d v}{d t}$, permite concluir que a velocidade $v$ do veículo deve obedecer à seguinte equação diferencial²:

$$
\frac{d v}{d t}+\frac{K}{m} v=g \cdot(\operatorname{sen} \theta-\mu \cos \theta)+\frac{F}{m},
$$

onde g é a aceleração da gravidade e $\theta$ a inclinação do plano em relação à horizontal. Não é difícil concluir que, neste caso, a velocidade limite ou terminal do objeto será dada por:

$$
v_{L}=\frac{m g}{K}(\operatorname{sen} \theta-\mu \cos \theta)+\frac{F}{K} \text {. }
$$

2 Entre tantas obras de referência que apresentam o equacionamento dinâmico de corpos sobre planos inclinados, o leitor interessado pode consultar com proveito o capítulo 5 de Halliday e Resnick (2016), bem como o capítulo 3 de Rade (2017). 
Fazendo $k=K / m$ e $\alpha=g(\operatorname{sen} \theta-\mu \cos \theta)$, a equação diferencial (1) assume a forma mais simples:

$$
\frac{d v}{d t}+k v=k \cdot v_{L}
$$

onde

$$
v_{L}=\frac{\alpha}{k}+\frac{F}{K}=\frac{1}{K}(m \alpha+F) .
$$

A equação diferencial (2) que modela a velocidade dos veículos é uma equação linear ${ }^{3}$. Admitindo que seja nula a velocidade inicial $[v(0)=0$ ], sua resolução fornece:

$$
v(t)=v_{L}\left(1-e^{-k t}\right)=\left(\frac{\alpha}{k}+\frac{F}{K}\right)\left(1-e^{-k t}\right) .
$$

\subsection{Equação de posição}

A equação de posição pode ser obtida por integração direta da equação de velocidade (4). Admitindo que seja nula a posição inicial $[s(0)=0]$, a integração fornece:

$$
s(t)=\int_{0}^{t} v(t) d t=v_{L}\left(t+\frac{e^{-k t}}{k}-\frac{1}{k}\right)=\left(\frac{\alpha}{k}+\frac{F}{K}\right)\left(t+\frac{e^{-k t}}{k}-\frac{1}{k}\right) .
$$

Vale notar que, depois de transcorrido um intervalo de tempo suficientemente longo, o termo exponencial torna-se desprezível frente à unidade: $e^{-k t}<<1$. Nessa situação, a velocidade tende ao valor constante da velocidade limite $v_{L}$ e a equação de posição pode ser aproximada pela equação de um movimento retilíneo uniforme:

$$
s(t) \simeq v_{L} \cdot t-\frac{v_{L}}{k}=v_{L} \cdot(t-m / K) .
$$

\subsection{Tempo para regime estacionário}

A partir do instante em que o objeto venha atingir $95 \%$ de sua velocidade limite ou terminal $v_{L}$, considera-se que teve início o regime estacionário, assim chamado porque, a partir de então, sua velocidade se manterá praticamente constante ${ }^{4}$. A imposição dessa condição na equação de

\footnotetext{
${ }^{3}$ Em Nagle, Saff e Snider (2012), o método de resolução das equações diferenciais lineares de primeira ordem é abordado na seção 2.3 (p. 35); nessa obra, a solução da equação de velocidade aqui apresentada pode ser vista nas p. 82-84. Em Zill e Cullen (2007), esse tópico pode ser encontrado na seção 2.5, equações lineares (p. 68).

${ }^{4} \mathrm{~A}$ análise do comportamento de grandezas que variam exponencialmente tendendo a um valor limite, bem como a distinção entre regime transiente e regime estacionário, é matéria comumente tratada nos livros de equações diferenciais. O leitor pode encontrar uma exposição da matéria em Nagel, Saff e Snider (2012), seção 3.4 - Mecânica newtoniana (p. 94). Por vezes, estipula-se que a grandeza deva atingir $98 \%$ ou 99\% de seu valor limite (correspondente a 4 ou 5 constantes de tempo) para ingressar em regime estacionário.
} 
velocidade (4) permite calcular o tempo necessário para que o objeto ingresse em regime estacionário, aqui denominado $T_{e}$ :

$$
\frac{95}{100} v_{L}=v_{L}\left(1-e^{-k T_{e}}\right) \Rightarrow \frac{19}{20}=1-e^{-k T_{e}}
$$

A resolução dessa última equação fornece:

$$
T_{e}=\frac{1}{k} \ln 20 \simeq \frac{3}{k}=\frac{3 m}{K}
$$

Interessante notar que $T_{e}$ depende apenas da constante $k=K / m$, e não da constante $\alpha$. Portanto, pode-se concluir que o tempo para atingir o regime estacionário independe dos valores do coeficiente de atrito de rolamento e da inclinação da rampa, mas apenas da relação entre a massa e a constante de arrasto aerodinâmico dos veículos.

\subsection{Extremando a função de posição}

Quando se considera que os móbiles não sejam dotados de força de tração e descem uma rampa apenas pela ação da força peso, é legítimo inferir que, em virtude do efeito provocado pela força de arrasto, quanto maior for a massa dos móbiles, maior será o desempenho, isto é, a distância percorrida em um intervalo fixo de tempo. Esse comportamento não se repete, contudo, quando se considera que os móbiles sejam dotados de propulsão própria. Como a aceleração é inversamente proporcional à massa, deve-se admitir que a ação da força de propulsão constante tenderá a produzir, inversamente, um desempenho tanto maior quanto menor for a massa dos veículos. Portanto, é razoável supor que, sob a ação conjunta dessas duas forças (propulsão e peso), possa haver um valor ótimo de massa capaz de maximizar o desempenho. De fato, como se verá a seguir, satisfeitas determinadas condições, a função de desempenho admite um extremante.

Procurando analisar a influência da variação da massa $m$ do veículo sobre a posição $S$, e, portanto, sobre a distância percorrida em um intervalo determinado de tempo $t$ (o tempo da competição), é preciso primeiramente obter a função de posição $s=s(m)$. Para tanto, note-se que é possível reescrever a função de posição (5) como sendo:

$$
s(k)=\frac{1}{K k^{2}}(F k+\alpha K)\left(k t+e^{-k t}-1\right)
$$

A substituição $k=K / m$ na equação (6) fornece:

$$
s(m)=\frac{1}{K^{2}}(F+m \alpha)\left[K t+m\left(e^{-\frac{K}{m} t}-1\right)\right]
$$


O estudo analítico do desempenho $s$ em função da variação da massa $m$ dos móbiles, realizado a seguir, considera constantes todos os demais parâmetros, inclusive o tempo $t$ estipulado para a competição.

A existência de um possível extremante para a função de posição $s(m)$, dada pela equação (7), está associada à existência de pelo menos uma raiz real para a equação5: $\frac{d s}{d m}=0$. Considerando que $s(m)=s(k(m))$, onde $k(m)$ corresponde à função $k=K / m$, a regra da cadeia para derivação de funções compostas permite o seguinte desenvolvimento:

$$
\frac{d s}{d m}=0 \Rightarrow \frac{d s}{d k} \frac{d k}{d m}=0 \Rightarrow-\frac{K}{m^{2}} \frac{d s}{d k}=0 \Rightarrow \frac{d s}{d k}=0 .
$$

Pode-se concluir, portanto, que é legítimo extremar a função $s(k)$, expressa na equação (6), em lugar da função $s(m)$, dada pela equação (7). Assim procedendo, obtém-se a equação:

$$
-2 k^{-3}(F k+\alpha K)\left(k t+e^{-k t}-1\right)+k^{-2} F\left(k t+e^{-k t}-1\right)+k^{-2} t(F k+\alpha K)\left(1-e^{-k t}\right)=0 .
$$

A procura de uma raiz para a equação (8) implica a resolução de uma equação transcendente em $k$, tarefa que exige o recurso a métodos numéricos. No presente trabalho, de cunho eminentemente analítico, não será esse o recurso empregado. Visando uma solução analítica, convém observar que, no caso em que $t \geq T_{e}$, os termos exponenciais tornam-se desprezíveis $\left(e^{-k t}<<1\right)$. Nesse caso, a equação transcendente (8) se reduz a uma equação algébrica de solução mais simples:

$$
-2 k^{-3}(F k+\alpha K)(k t-1)+k^{-2} F(k t-1)+k^{-2} t(F k+\alpha K)=0
$$

A adoção da equação (9) possibilita a seguinte resolução para a equação do extremante:

$$
\begin{gathered}
-2 k^{-3}(F k+\alpha K)(k t-1)+k^{-2} F(k t-1)+k^{-2} t(F k+\alpha K)=0 \Rightarrow \\
-2(F k+\alpha K)(k t-1)+k F(k t-1)+k t(F k+\alpha K)=0 \Rightarrow \\
(-F k-2 \alpha K)(k t-1)+k t(F k+\alpha K)=0 \Rightarrow \\
(-F k-2 \alpha K) k t+(F k+\alpha K) k t+F k+2 \alpha K=0 \Rightarrow \\
F k-\alpha K k t+2 \alpha K=0 \Rightarrow k(F-\alpha K t)+2 \alpha K=0 \Rightarrow \\
k=\frac{2 \alpha K}{\alpha K t-F}
\end{gathered}
$$

Como $k=K / m$, sua substituição na equação (10) fornece:

\footnotetext{
${ }^{5} \mathrm{~A}$ aplicação das derivadas na resolução dos problemas de otimização é tópico que integra a imensa maioria das obras de Cálculo Diferencial e Integral. Em Thomas, Weir e Hass (2011), pode ser vista na seção 3.5 - Modelagem e otimização (p. 272).
} 


$$
\begin{gathered}
\frac{K}{m}=\frac{2 \alpha K}{\alpha K t-F} \Rightarrow m=\frac{\alpha K t-F}{2 \alpha} \Rightarrow \\
m=\frac{1}{2}(K t-F / \alpha)
\end{gathered}
$$

A expressão (11) permite calcular o valor da massa $m$ que maximiza o desempenho do veículo (isto é, a distância percorrida no intervalo de tempo $t$ da competição) em função dos parâmetros previamente definidos, quais sejam: a força de tração $F$, a constante de arrasto $K$ e a constante $\alpha$, associada ao coeficiente de atrito de rolamento e à inclinação da rampa, segundo a fórmula $\alpha=g(\operatorname{sen} \theta-\mu \cos \theta)$.

Uma consideração adicional precisa ser feita quanto à validade da expressão (11). Primeiramente, convém lembrar que ela resulta de uma simplificação da equação transcendente (8), em que os termos exponenciais puderam ser desprezados. Essa simplificação só é válida nas situações em que o tempo estabelecido para competição for maior que o tempo para regime estacionário, isto é, em que $t>T_{e} \Rightarrow t>3 / k$. Caso se explicite $F$ da equação (11), obtém-se: $F=\alpha(K t-2 m)$. A expressão mostra que se deve ter $K t>2 m \Rightarrow t>2 / k$ para que $F$ seja positivo. Como $3 / k>2 / k$, pode-se concluir que basta tomar $t>T_{e}$ para que esta condição se verifique.

Além disso, é preciso observar que a função $s(m)$, dada pela equação (7), nem sempre apresenta um ponto de máximo. Com efeito, a presença de um ponto de máximo exige que os valores estabelecidos para os parâmetros $F, \alpha, K$ e $t$ satisfaçam determinadas condições. A esse respeito, observando a equação (11), é forçoso constatar que, como o valor de $m$ deve ser positivo: $K t>F / \alpha \Rightarrow F<\alpha K t$. A existência do máximo encontrado para a posição $s$ exige, portanto, que a força de tração $F$ seja menor que certo valor máximo. Como se verá nas páginas subsequentes, é preciso ainda que esta força $F$ seja maior que um determinado valor mínimo. Assim sendo, o extremante fornecido pela equação (11) só se verifica quando a força de tração $F$ se encontra dentro de um intervalo de variação, o qual será definido mais adiante.

\subsection{Cálculo do valor máximo da função de posição}

Como determinado na equação (10), o ponto de máximo da função de posição ocorre para $k_{M}=\frac{2 \alpha K}{\alpha K t-F}$. Assim sendo, o valor máximo da função será dado por $s\left(k_{M}\right)$, em $s(k)$ é expresso pela equação (6). No entanto, para $t \geq T_{e}$, tem-se que $e^{-k t}<<1$, fazendo com que a equação (6) assuma a forma mais simples: 


$$
s(k) \simeq \frac{1}{K k^{2}}(F k+\alpha K)(k t-1) .
$$

Empregando a equação (12) para cálculo do valor máximo da função de posição, vem:

$$
\begin{gathered}
s_{\text {máx }}=s\left(k_{M}\right) \simeq \frac{1}{K k_{M}{ }^{2}}\left(F k_{M}+\alpha K\right)\left(k_{M} t-1\right) \Rightarrow \\
s_{\text {máx }} \simeq \frac{1}{K}\left(\frac{\alpha K t-F}{2 \alpha K}\right)^{2}\left(\frac{2 \alpha K F}{\alpha K t-F}+\alpha K\right)\left(\frac{2 \alpha K t}{\alpha K t-F}-1\right) \Rightarrow \\
s_{\text {máx }} \simeq \frac{1}{K}\left(\frac{\alpha K t-F}{2 \alpha K}\right)^{2}\left(\frac{\alpha K F+\alpha^{2} K^{2} t}{\alpha K t-F}\right)\left(\frac{\alpha K t+F)}{\alpha K t-F}\right) \Rightarrow \\
s_{\text {máx }} \simeq \frac{\alpha(\alpha K t+F)^{2}}{(2 \alpha K)^{2}}=\frac{1}{4 \alpha K^{2}}(\alpha K t+F)^{2} \Rightarrow \\
s_{\text {máx }} \simeq \frac{1}{4 \alpha}(\alpha t+F / K)^{2}=\frac{\alpha}{4 K^{2}}(K t+F / \alpha)^{2} .
\end{gathered}
$$

\subsection{A função de posição e seus limites}

Tem interesse calcular o valor dos limites $\lim _{m \rightarrow 0^{+}} s(m)$ e $\lim _{m \rightarrow \infty} s(m)$, pois expressam os valores para os quais tende a posição (e, portanto, a distância percorrida no intervalo de tempo da competição) quando a massa $m$ dos móbiles tende a assumir valores muito pequenos $\left(m \rightarrow 0^{+}\right)$ ou valores extremamente grandes $(m \rightarrow \infty)$.

O cálculo do limite lateral à direita de zero pode ser feito de forma imediata a partir da equação (7):

$$
\lim _{m \rightarrow 0^{+}} s(m)=\frac{1}{K^{2}} \lim _{m \rightarrow 0^{+}}(F+m \alpha)\left[K t+m\left(e^{-\frac{K}{m} t}-1\right)\right]=\frac{F K t}{K^{2}}=\frac{F t}{K} .
$$

Diferentemente do anterior, porém, o limite no infinito da função de posição $s(m)$ não se faz de forma imediata, pois assume uma forma indeterminada. Como a constante $K$ é inversamente proporcional à massa $m$, pode-se concluir que quando $m \rightarrow \infty, k \rightarrow 0$. É legítimo, portanto, calcular o $\lim _{k \rightarrow 0^{+}} s(k)$ em lugar do $\lim _{m \rightarrow \infty} s(m)$ procurado. Servindo-se da equação (6), tem-se que:

$$
\lim _{k \rightarrow 0^{+}} s(k)=\frac{1}{K} \lim _{k \rightarrow 0^{+}}\left[F t+F \frac{\left(e^{-k t}-1\right)}{k}+\frac{\alpha K t}{k}+\alpha K \frac{\left(e^{-k t}-1\right)}{k^{2}}\right] .
$$

A expansão em Série de Maclaurin dos termos exponenciais que aparecem na equação (15) fornece: 


$$
\begin{aligned}
& \frac{\left(e^{-k t}-1\right)}{k}=-t+\frac{k t^{2}}{2}-\frac{k^{2} t^{3}}{6}+\ldots \\
& \frac{\left(e^{-k t}-1\right)}{k^{2}}=-\frac{t}{k}+\frac{t^{2}}{2}-\frac{k t^{3}}{6}+\ldots
\end{aligned}
$$

Substituindo as expansões em série de potência na equação (15), obtém-se:

$$
\lim _{k \rightarrow 0^{+}} s(k)=\frac{1}{K} \lim _{k \rightarrow 0^{+}}\left[F t+F\left(-t+\frac{k t^{2}}{2}-\frac{k^{2} t^{3}}{6}+\ldots\right)+\frac{\alpha K t}{k}+\alpha K\left(-\frac{t}{k}+\frac{t^{2}}{2}-\frac{k t^{3}}{6}+\ldots\right)\right]=\frac{\alpha t^{2}}{2}
$$

Portanto, pode-se concluir que:

$$
\lim _{m \rightarrow \infty} s(m)=\lim _{k \rightarrow 0^{+}} s(k)=\frac{\alpha t^{2}}{2} .
$$

O gráfico da Figura 2 apresenta a forma típica do comportamento da curva de desempenho $s$ em função da massa $m$ dos móbiles, quando a função admite um extremo. Podese observar que a distância percorrida parte de $s_{0}$, quando $m$ tende a zero, passa por um valor máximo $s_{\text {máx }}$, que se dá para $m=(K t-F / \alpha) / 2$, e depois decai tendendo ao valor assintótico $s_{L}$, à medida que $m$ tende a assumir valores infinitamente grandes.

Figura 2 - Gráfico da posição $s$ (ordenada) em função da massa $m$ (abscissa). Curva revela existência de um extremante.

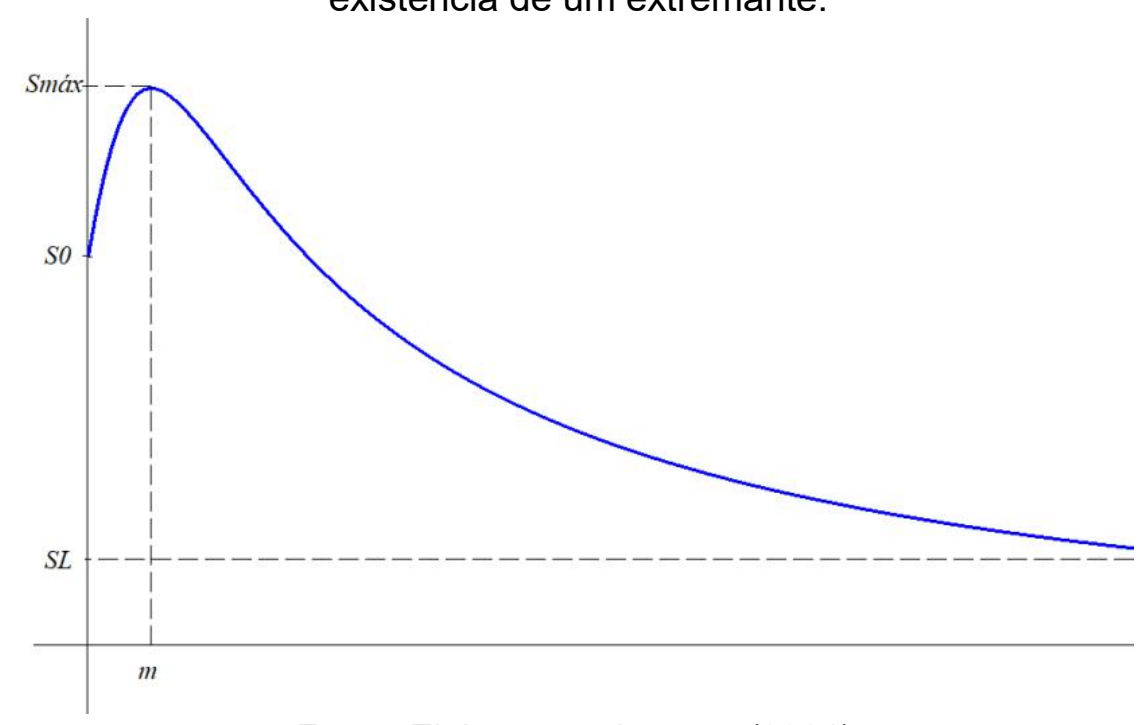

Fonte: Elaboração do autor (2021).

Conforme calculado anteriormente, em conformidade com as equações (11), (13), (14) e (16), os valores característicos da curva de posição delineada na Figura 2 são dados pelas seguintes expressões:

$$
m=\frac{1}{2}(K t-F / \alpha) ; s_{\text {máx }} \simeq \frac{1}{4 \alpha}(\alpha t+F / K)^{2} ; s_{0}=\lim _{m \rightarrow 0^{+}} s(m)=\frac{F t}{K} ; s_{L}=\lim _{m \rightarrow \infty} s(m)=\frac{\alpha t^{2}}{2} .
$$




\subsection{Velocidade limite e potência máxima para desempenho ótimo}

No caso em que a massa $m$ assume o valor que maximiza o desempenho, dado pela equação (11), a expressão (3) para a velocidade limite $v_{L}$ assume a forma:

$$
\begin{gathered}
v_{L}=\frac{1}{K}(m \alpha+F)=\frac{\alpha}{K} m+\frac{F}{K}=\frac{\alpha}{2 K}(K t-F / \alpha)+\frac{F}{K} \Rightarrow \\
v_{L}=\frac{1}{2}(\alpha t+F / K) .
\end{gathered}
$$

De um eventual motor, que deverá fornecer a força de tração constante $F$ ao veículo, será exigida uma potência tanto maior quanto maior for sua velocidade, e que tenderá a ser máxima quanto mais próxima da velocidade limite. Portanto, se chamarmos de $P$ essa potência máxima, ela pode ser calculada a partir da velocidade limite $v_{L}$ :

$$
P=F \cdot v_{L}=\frac{F}{2}(\alpha t+F / K)
$$

Pode-se ainda expressar a potência $P$ em termos da massa ótima $m$. Lembrando a expressão obtida da equação (11), segundo a qual $F=\alpha(K t-2 m)$, sua substituição na equação (17) fornece:

$$
\begin{gathered}
P=\frac{F}{2}(\alpha t+F / K)=\frac{\alpha}{2}(K t-2 m)[\alpha t+\alpha(t-2 m / K)]=\frac{\alpha^{2}}{2}(K t-2 m)(2 t-2 m / K) \Rightarrow \\
P=\frac{\alpha^{2}}{K}(K t-2 m)(K t-m) .
\end{gathered}
$$

\subsection{Sensibilidade do desempenho à variação dos parâmetros}

Interessa investigar qual a sensibilidade do desempenho (distância máxima percorrida) à variação dos parâmetros $K$ e $\mu$. É possível obter uma boa estimativa da variação absoluta do desempenho por meio do cálculo da diferencial total $d s_{\text {máx }}$, segundo expressão:

$$
d s_{\text {máx }}=\frac{\partial s_{\max }}{\partial K} d K+\frac{\partial s_{\max }}{\partial \mu} d \mu .
$$

O cálculo das derivadas parciais que compõem a equação (18) realizado a partir da equação (13) fornece:

$$
\begin{gathered}
{\left[s_{\text {máx }}\right]_{K}=\frac{\partial s_{\text {máx }}}{\partial K}=-\frac{F}{2 \alpha K^{2}}(\alpha t+F / K)=-\frac{F}{2 K^{3}}(K t+F / \alpha)} \\
{\left[s_{\text {máx }}\right]_{\mu}=\frac{\partial s_{\text {máx }}}{\partial \mu}=\frac{\partial s_{\text {máx }}}{\partial \alpha} \frac{\partial \alpha}{\partial \mu}=-g \cos \theta \frac{\partial s_{\text {máx }}}{\partial \alpha}=-\frac{g \cos \theta}{4 K^{2}}(K t-F / \alpha)^{2}}
\end{gathered}
$$


em que se empregou o resultado:

$$
\frac{\partial s_{\max }}{\partial \alpha}=\frac{1}{4 \alpha^{2}}(\alpha t-F / K)^{2}=\frac{1}{4 K^{2}}(K t-F / \alpha)^{2}=(m / K)^{2} .
$$

Como é mais significativo, não o valor da variação absoluta do desempenho, mas o de sua variação percentual relativamente ao valor de $s_{\text {máx }}$, dado pela equação (13), seria interessante calcular os seguintes quocientes, que fornecem a variação percentual relativa do desempenho:

$$
\begin{gathered}
\frac{\left[s_{\text {máx }}\right]_{K}}{s_{\text {máx }}} d K \times 100 \%=-\frac{2 F}{(\alpha K t+F)} \frac{d K}{K} \times 100 \% \\
\frac{\left[s_{\text {máx }}\right]_{\mu}}{s_{\text {máx }}} d \mu \times 100 \%=-\left(\frac{\alpha K t-F}{\alpha K t+F}\right)^{2} \frac{d \mu}{\operatorname{tg} \theta-\mu} \times 100 \%
\end{gathered}
$$

Lembrando que, segundo a equação (11), $\alpha K t-F=2 \alpha m$, as expressões para sensibilidade do desempenho podem ser reescritas em termos do valor da massa $m$, que otimiza o desempenho, da seguinte forma:

$$
\begin{gathered}
\frac{\left[s_{\text {máx }}\right]_{K}}{s_{\text {máx }}} d K \times 100 \%=-\frac{F}{(\alpha m+F)} \frac{d K}{K} \times 100 \% \\
\frac{\left[s_{\text {máx }}\right]_{\mu}}{s_{\text {máx }}} d \mu \times 100 \%=-\left(\frac{\alpha m}{\alpha m+F}\right)^{2} \frac{d \mu}{\operatorname{tg} \theta-\mu} \times 100 \%
\end{gathered}
$$

A expressão (19) mostra que, relativamente à constante de arrasto $K$, a sensibilidade do ganho de desempenho será tanto maior quanto menor for a massa $\mathrm{m}$. Isto significa que, sendo a massa do veículo relativamente pequena, reduções na constante de arrasto $K$ poderão acarretar grandes ganhos de desempenho.

Já relativamente à constante de atrito de rolamento $\mu$, a expressão (20) mostra que a sensibilidade do desempenho aumenta com a massa $m$. Quanto maior for a massa do veículo, maiores deverão ser os ganhos de desempenho com uma redução obtida neste coeficiente. Para valores de massa muito pequenos, vale notar que a sensibilidade tende a ser praticamente nula, de modo que reduções obtidas neste coeficiente não trarão ganho significativo de desempenho.

\subsection{Algumas curvas ilustrativas de desempenho}

As curvas de desempenho apresentadas a seguir foram traçadas empregando-se a função $s(m)$ dada pela equação (7), que expressa como varia o desempenho $s$ (distância percorrida no intervalo de tempo $t$ estipulado pela competição) em função da variação da massa $m$ dos móbiles. Em todos os gráficos, a distância $s$ (em metros) está disposta no eixo das ordenadas e a massa $m$ (em kg) no eixo das abscissas. 
i) Nas curvas de desempenho apresentadas na Figura 3, foram mantidos constantes os parâmetros $\alpha=1 / 2, F=15 \mathrm{~N}$ e $t=50$ segundos, fazendo-se variar a constante de arrasto $K$. Como se pode observar, as curvas revelam sensibilidade maior do desempenho às variações da constante $K$ quanto menor é a massa $m$ dos móbiles. A sensibilidade aumenta intensamente para valores de massa que tendem a ser muito pequenos. Em contrapartida, quando a massa se torna muito grande, a sensibilidade tende a ser praticamente nula.

Figura 3 - Curvas da posição $s$ (ordenada) em função da massa $m$ (abscissa)

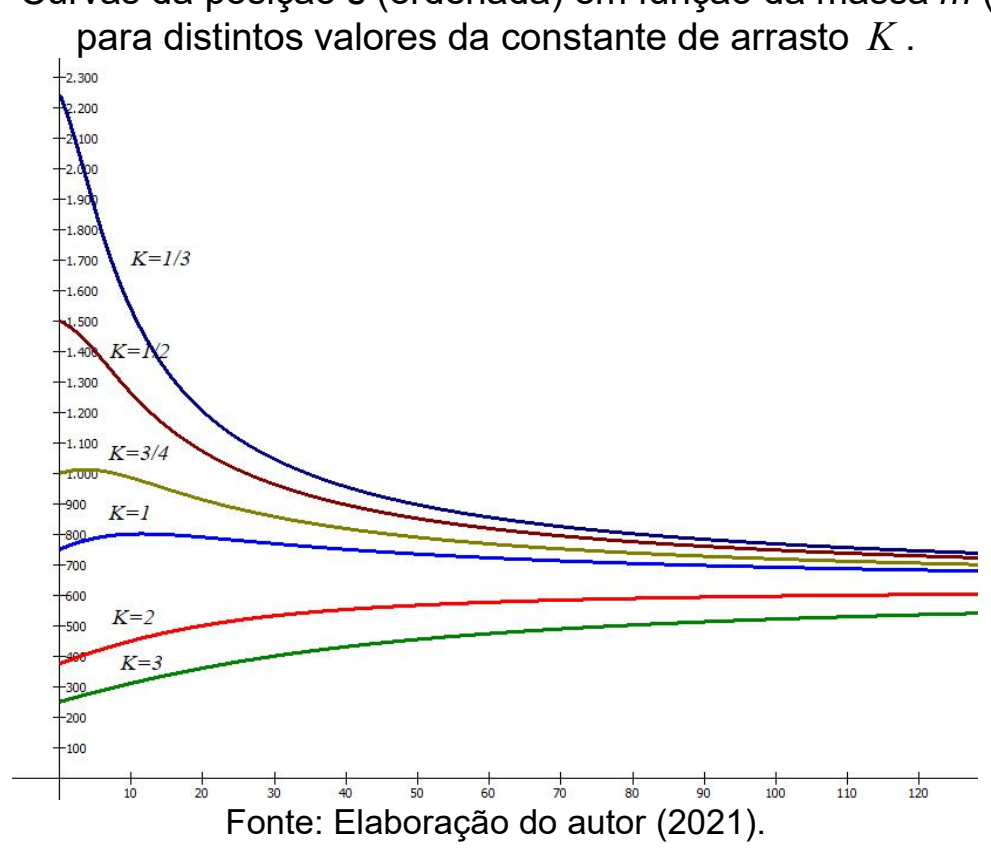

ii) Nas curvas de desempenho apresentadas na Figura 4, foram mantidos constantes os parâmetros $K=1 \mathrm{~N} . \mathrm{s} / \mathrm{m}, F=15 \mathrm{~N}$ e $t=50$ segundos, fazendo-se variar a constante $\alpha=g(\operatorname{sen} \theta-\mu \cos \theta)$.

Figura 4 - Curvas da posição $s$ (ordenada) em função da massa $m$ (abscissa) para distintos valores da constante $\alpha$.

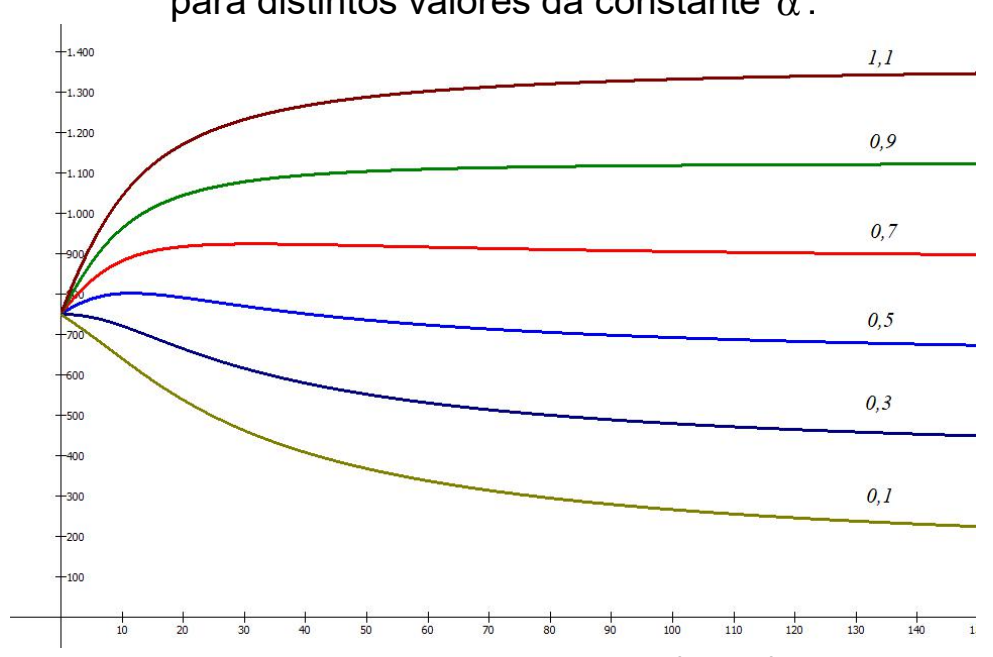

Fonte: Elaboração do autor (2021). 
Como se pode constatar, as curvas da Figura 4 revelam sensibilidade maior do desempenho às variações de $\alpha$ quanto maior é a massa $m$ dos móbiles. Quando a massa se torna muito pequena, a sensibilidade tende a ser praticamente nula.

\subsection{Considerações sobre a força de tração}

Sensibilidade do desempenho relativamente a variações da força de tração. Como não se pode garantir, na prática, que o valor real da força de tração seja exatamente aquele especificado, é interessante investigar a sensibilidade do desempenho relativamente a eventuais variações na força de tração. Uma estimativa da variação $d s$ do desempenho ocasionada por pequenas variações incrementais $d F$ na força de tração pode ser obtida por meio de diferenciais, segundo a expressão:

$$
d s_{\text {max }}=\frac{\partial s_{\max }}{\partial F} d F
$$

O cálculo da derivada parcial presente na expressão (21), realizado a partir da equação (13), leva ao seguinte resultado para a variação percentual relativa do desempenho:

$$
\begin{gathered}
{\left[s_{\text {máx }}\right]_{F}=\frac{\partial s_{\text {máx }}}{\partial F}=\frac{1}{2 \alpha K}(\alpha t+F / K)=\frac{1}{2 K^{2}}(K t+F / \alpha)} \\
\frac{\left[s_{\text {máx }}\right]_{F}}{s_{\text {máx }}} d F \times 100 \%=\frac{2 . d F}{\alpha K t+F} \times 100 \%=\frac{d F}{\alpha m+F} \times 100 \%
\end{gathered}
$$

A expressão (22) atesta que a sensibilidade do desempenho relativamente à força de tração $F$ é inversamente proporcional à massa $m$, isto é, quanto maior for a massa $m$, menor será a variação percentual de ganho de desempenho com variações da força de tração $F$.

Figura 5 - Curvas da posição $s$ (ordenada) em função da massa $m$ (abscissa) para distintos valores da força de propulsão $F$.

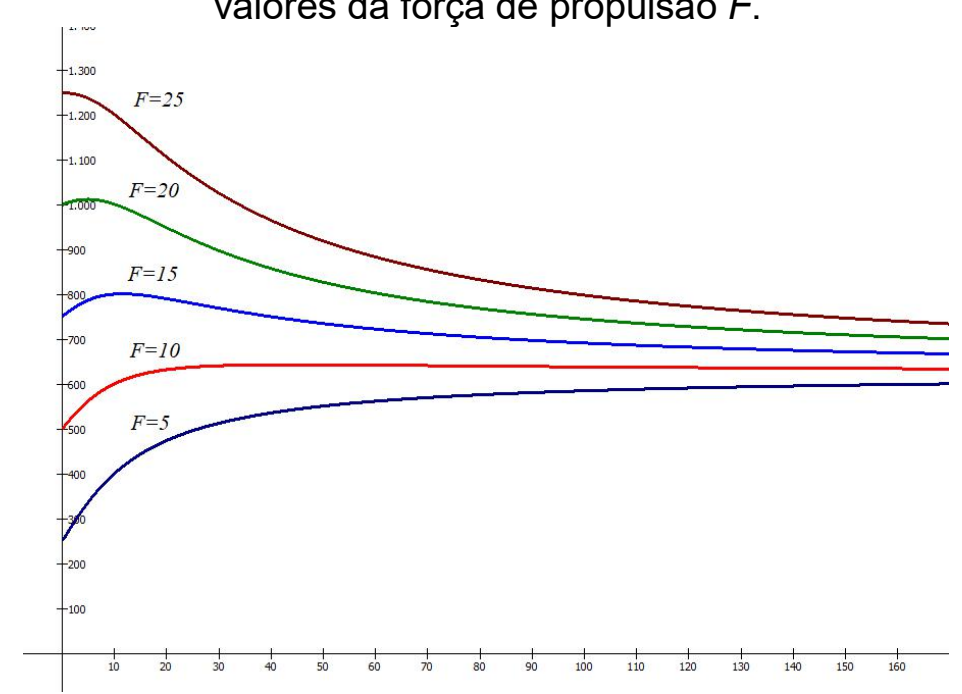

Fonte: Elaboração do autor (2021). 
Esse comportamento pode ser verificado nas curvas de desempenho traçadas na Figura 5, nas quais se empregou a função $s(m)$ dada pela equação (7). Foram mantidos constantes os parâmetros $K=1 \mathrm{~N} . \mathrm{s} / \mathrm{m} ; \alpha=1 / 2$ e $t=50$ segundos, fazendo-se variar a força de tração $F$. Vale observar que as curvas revelam possuir um extremante apenas para $10<F<25$. Essa faixa de variação de $F$ como condição de existência do extremante será explicitada logo a seguir.

Considerações sobre a condição de existência do extremante. Uma análise do gráfico apresentado na Figura 2 permite a seguinte asserção: para que o ponto de máximo da função $s(m)$ encontrado anteriormente delineie efetivamente um máximo, é forçoso que o valor máximo da função, $s_{\text {máx }}$, seja não só maior que $s_{L}$ (o valor do limite no infinito), como também maior que $s_{0}$ (o valor do limite lateral à direita do zero). Recorrendo às equações (13), (14) e (16) e impondo as duas condições mencionadas, obtém-se

i) $S_{\text {máx }}>S_{L}$ :

$$
\frac{1}{4 \alpha}(\alpha t+F / K)^{2}>\frac{\alpha t^{2}}{2} \Rightarrow(\alpha K t+F)^{2}>2(\alpha K t)^{2}
$$

Fazendo $\alpha K t=z,(F+z)^{2}>2 z^{2} \Rightarrow z^{2}-2 F z-F^{2}<0$.

Esta inequação em $z$ tem como solução o intervalo: $F(1-\sqrt{2})<z<F(1+\sqrt{2})$. Como, no entanto, $z=\alpha K t>0$, a solução procurada se torna: $0<\alpha K t<F(1+\sqrt{2})$. Portanto, a expressão estabelece, como condição de existência do extremo, um valor mínimo para a força $F$ :

$$
F>\frac{\alpha K t}{1+\sqrt{2}} \Rightarrow F>(\sqrt{2}-1) \cdot \alpha K t \text {. }
$$

ii) $S_{\text {máx }}>s_{0}$ :

$$
\frac{1}{4 \alpha}(\alpha t+F / K)^{2}>\frac{F t}{K} \Rightarrow(\alpha K t+F)^{2}>4 F(\alpha K t) .
$$

Fazendo novamente $\alpha K t=z$, vem:

$$
(F+z)^{2}>4 F z \Rightarrow z^{2}-2 F z+F^{2}>0 \Rightarrow(z-F)^{2}>0 .
$$

Esta inequação em $z$ tem como solução todo real, com exceção de $z=F \therefore F \neq \alpha K t$. Contudo, já havia sido estabelecido anteriormente, para garantir que o valor da massa $m$ fosse positivo, que $F<\alpha K t$. Portanto, pode-se concluir que os valores da força de tração $F$ devem situar-se no seguinte intervalo:

$$
(\sqrt{2}-1) . \alpha K t<F<\alpha K t
$$


Esta é a condição que deve ser satisfeita para que a função $s(m)$ admita um extremante; consequentemente, é também a condição de validade da equação (11), que fornece o valor de massa que maximiza o desempenho.

Para valores de $F$ menores, tais que $F<(\sqrt{2}-1) . \alpha K t$, não ocorre extremante. Nesse caso, quanto maior for a massa dos veículos, maior será a distância percorrida, sendo o limite da distância dado por: $s_{L}=\lim _{m \rightarrow \infty} s=\frac{\alpha t^{2}}{2}$. Essa situação, seria oportuno observar, contempla o caso de móbiles sem força de tração $(F=0)$ que serão estudados na seção seguinte deste trabalho.

Para valores de $F$ maiores, tais que $F>\alpha K t$, também não ocorre extremante. Curiosamente, porém, neste caso, quanto menor for a massa dos veículos, maior será a distância percorrida, sendo o limite da distância dado por: $s_{0}=\lim _{m \rightarrow 0^{+}} s=\frac{F t}{K}$. Nesta situação, portanto, o desafio em uma competição seria conseguir fabricar os móbiles mais leves possíveis, sem prejuízo de sua resistência estrutural.

\subsection{Roteiro para definição dos termos da competição}

Em uma competição com veículos dotados de propulsão, seria interessante que houvesse um valor específico de massa dos veículos capaz de proporcionar o máximo desempenho. Seria atribuição de cada equipe competidora, na etapa de projeto, procurar determinar o valor ótimo de massa a partir dos parâmetros de arrasto e atrito de rolamento de seu veículo encontrados experimentalmente. Ora, para que a função de posição admita extremante é preciso, como se mostrou há pouco, que a força de tração $F$ se encontre no intervalo $(\sqrt{2}-1) . \alpha K t<F<\alpha K t$. Este poderia ser, portanto, um critério prévio a ser observado para definição da força de tração. Além disso, como seria interessante que o processo de otimização trouxesse ganhos significativos, poder-se-ia adotar para a força $F$ um valor que assegure um extremo saliente para função distância percorrida $s(m)$. Para tanto, o valor da força $F$ poderia ser definido como ponto médio do intervalo. Isso significa fazer $F=\frac{\sqrt{2}}{2} \alpha K t$. Neste caso, a distância máxima percorrida no intervalo de tempo da competição (obtido para um valor otimizado de massa dos veículos) pode ser calculado a partir da equação (13), e será dada por:

$$
\begin{gathered}
s_{\text {máx }}=\frac{1}{4 \alpha K^{2}}(\alpha K t+F)^{2}=\frac{1}{4 \alpha K^{2}}\left(\alpha K t+\frac{\sqrt{2}}{2} \alpha K t\right)^{2} \Rightarrow \\
s_{\text {máx }}=\alpha t^{2}\left(1+\frac{\sqrt{2}}{2}\right)^{2}=(3 / 2+\sqrt{2}) \alpha t^{2} \simeq 2,91 . \alpha t^{2}
\end{gathered}
$$


Isto posto, e considerando que se dispõe, para realização da competição, de uma rampa retilínea com inclinação constante $\theta$ em relação à horizontal e comprimento total $L$, o seguinte roteiro poderia ser sugerido para definição dos critérios a serem adotados:

i) Em primeiro lugar, seria preciso obter o valor médio da constante de arrasto $\bar{K}$ de todos os veículos participantes, assim como o valor médio $\bar{\mu}$ dos coeficientes de atrito de rolamento da totalidade dos veículos competidores; de posse desse valor, calcular o valor médio $\bar{\alpha}=g(\operatorname{sen} \theta-\bar{\mu} \cos \theta)$.

ii) Em seguida, pode-se estipular o intervalo de tempo $t$ da competição a partir do comprimento $L$ da rampa disponível. Se, por simplicidade, fizermos $s_{\text {máx }}=3 \alpha t^{2}$, então:

$$
s_{\text {máx }}=L \Rightarrow 3 \bar{\alpha} t^{2}=L \Rightarrow t=\sqrt{\frac{L}{3 \bar{\alpha}}} \simeq 0,58 \sqrt{L / \bar{\alpha}}
$$

ii) Por fim, segundo as considerações feitas anteriormente, pode-se definir o valor da força de tração $F$ dos veículos a partir da expressão:

$$
F=\frac{\sqrt{2}}{2} \bar{\alpha} \bar{K} t=\bar{K} \sqrt{\frac{\bar{\alpha} L}{6}}
$$

Com isso, a potência máxima dos motores poderá ser calculada pela equação (17), a qual fornece:

$$
P=F(\bar{\alpha} t+F / \bar{K}) / 2=\bar{\alpha} \bar{K}(L+t \sqrt{6 \bar{\alpha} L}) / 6=\bar{\alpha} \bar{K} L(1+\sqrt{2}) / 6
$$

O cálculo do valor ótimo de massa que cada um dos veículos competidores deverá possuir é o desafio de projeto a ser enfrentado por cada equipe participante, o qual poderá ser efetuado empregando a equação (11) a partir dos valores específicos das constantes $K$ e $\alpha$ obtidos experimentalmente para seus veículos. Convém observar, porém, que cada equipe deverá ainda envidar esforços visando reduzir a constante de arrasto e o coeficiente de atrito de rolamento de seus móbiles, uma vez que a redução desses parâmetros acarreta ganho de desempenho. Assim sendo, cada alteração obtida no valor das constantes $K$ e $\alpha$ exigirá que o valor ótimo de massa seja recalculado e implementado na prática, reiteradamente. Mesmo concedendo que cada equipe participante deve possuir total liberdade para escolher, segundo o critério que se mostrar mais acertado, o valor da massa total de seus veículos, é possível antecipar, servindo-se da equação (11), que o valor médio da massa dos veículos participantes deverá oscilar em torno de:

$$
\bar{m}=\frac{1}{2 \bar{\alpha}}(\bar{\alpha} \bar{K} t-F)=\frac{1}{2}\left(1-\frac{\sqrt{2}}{2}\right) \bar{K} t \simeq \frac{3}{20} \bar{K} t=\frac{\sqrt{3}}{20} \bar{K} \sqrt{L / \bar{\alpha}}
$$

Isso porque deve-se admitir que a totalidade dos competidores procurará determinar a massa de seus veículos de modo a otimizar o desempenho. 


\subsection{Algumas curvas ilustrativas}

As curvas de desempenho apresentadas a seguir foram traçadas empregando-se a função $s(m)$ dada pela equação (7). Em todos os gráficos, a distância $s$ (em metros) está disposta no eixo das ordenadas e a massa $m(\mathrm{em} \mathrm{kg}$ ) no eixo das abscissas.

i) Para a curva de desempenho apresentada na Figura 6 foram utilizados os seguintes parâmetros: $K=1 \mathrm{~N} . \mathrm{s} / \mathrm{m}$; $\alpha=1 / 2$ e $F=15 \mathrm{~N}, t=50$ segundos, que implicam velocidade limite $v_{L}=(\alpha t+F / K) / 2=20 \mathrm{~m} / \mathrm{s}$. Neste caso, $k t=5$, valor que satisfaz a condição de regime estacionário $k t>3$.

O valor máximo do desempenho ocorre para um valor de massa próximo de $m=(K t-F / \alpha) / 2=10 \mathrm{~kg}$, enquanto o desempenho máximo correspondente é: $s_{\text {máx }} \simeq(\alpha t+F / K)^{2} / 4 \alpha=800$ metros. Demais valores característicos da curva de desempenho: $s_{0}=F t / K=750$ metros; $s_{L}=\alpha t^{2} / 2=625$ metros. A potência máxima exigida do motor será: $P=F(\alpha t+F / K) / 2=300 \mathrm{~W}$.

Figura 6 - Gráfico da posição $s$ (ordenada) em função da massa $m$ (abscissa). Ponto de máximo vizinho a $m=10 \mathrm{~kg}$.

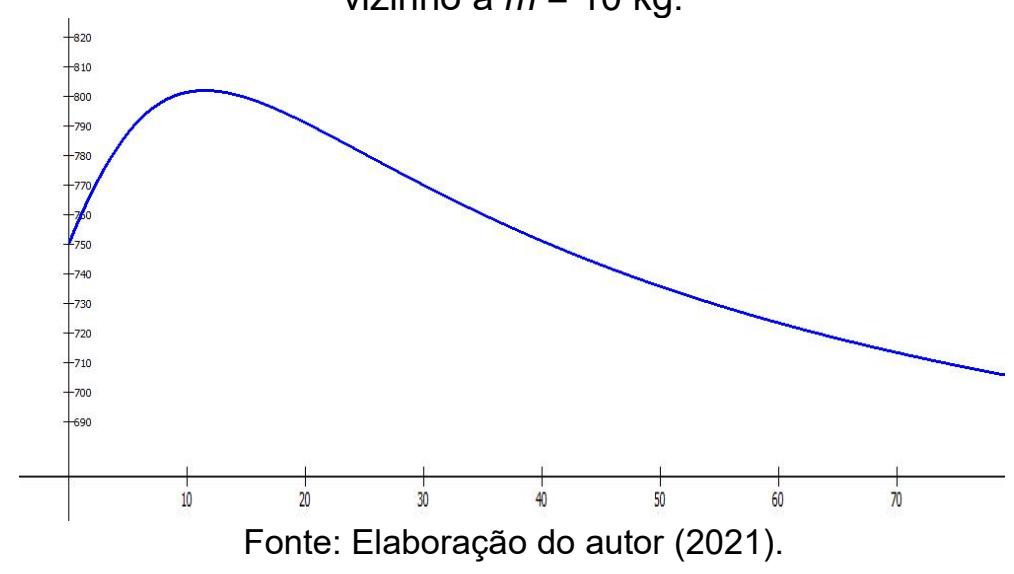

ii) No traçado da curva de desempenho apresentada na Figura 7 foram utilizados os seguintes parâmetros: $K=1 / 5 \mathrm{~N} . \mathrm{s} / \mathrm{m} ; \alpha=1 / 4$ e $F=1 \mathrm{~N}, t=40$ segundos, que implicam velocidade limite $v_{L}=(\alpha t+F / K) / 2=15 / 2=7,5 \mathrm{~m} / \mathrm{s}$. Neste caso, $k t=4$, valor que satisfaz a condição de regime estacionário $k t>3$.

O valor máximo do desempenho ocorre para um valor de massa próximo de $m=(K t-F / \alpha) / 2=2 \mathrm{~kg}$, enquanto o desempenho máximo correspondente é: $s_{\text {máx }} \simeq(\alpha t+F / K)^{2} / 4 \alpha=225$ metros. Demais valores característicos da curva de desempenho: $s_{0}=F t / K=200$ metros; $s_{L}=\alpha t^{2} / 2=200$ metros. A potência máxima exigida do motor será: $P=F(\alpha t+F / K) / 2=15 / 2=7,5 \mathrm{~W}$. 
Figura 7 - Gráfico da posição $s$ (ordenada) em função da massa $m$ (abscissa). Ponto de máximo vizinho a $m=3 \mathrm{~kg}$.

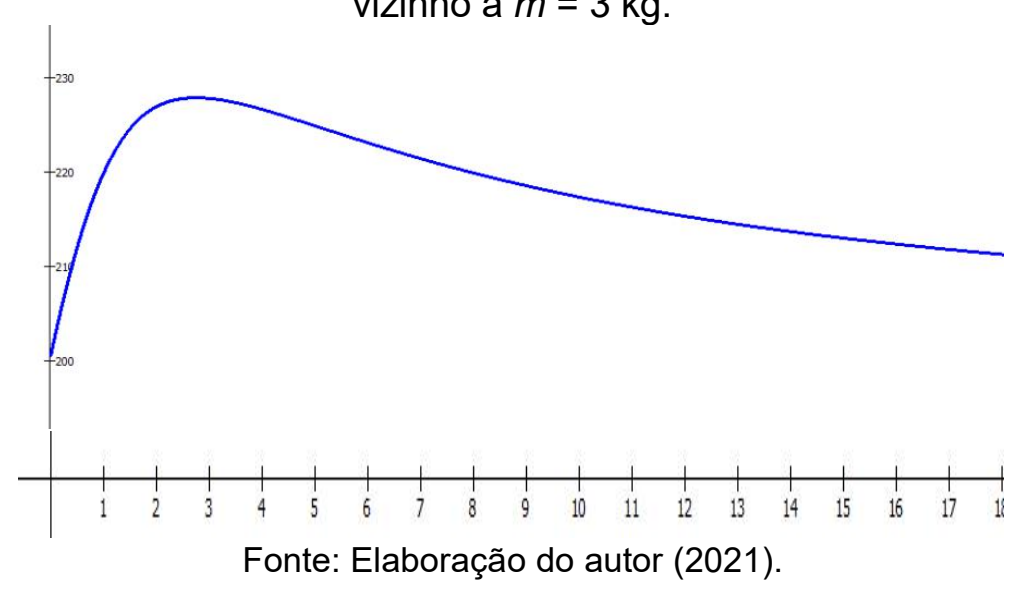

iii) Para a curva de desempenho apresentada na Figura 8 foram empregados os seguintes parâmetros: $K=1 / 5 \mathrm{~N} . \mathrm{s} / \mathrm{m} ; \alpha=1 / 4 ; F=5 / 8 \mathrm{~N}$ e $t=20$ segundos. Neste caso, $k t \simeq 4 / 3$, valor que não satisfaz a condição de regime estacionário, que exige $k t>3$. Note-se que ponto de máximo observado na curva ocorre para um valor bastante distinto do fornecido pela equação (11), que é: $m=(K t-F / \alpha) / 2=13 / 4=3,25 \mathrm{~kg}$. A velocidade limite, portanto, não seria atingida no tempo da competição para este valor de massa.

Figura 8 - Gráfico da posição $s$ (ordenada) em função da massa $m$ (abscissa). Ponto de máximo ocorre para $m \simeq 0,85 \mathrm{~kg}$.

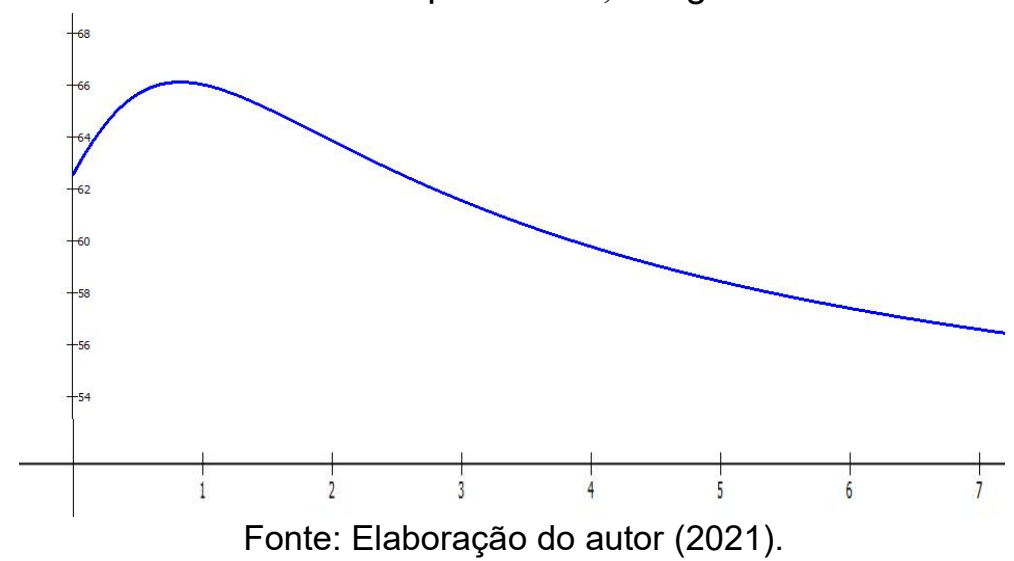

\section{Análise de desempenho para móbiles sem força de propulsão}

Considere-se agora o caso de móbiles que não sejam dotados de força de propulsão própria e que descem uma rampa inclinada, a partir do repouso, graças à ação única e exclusiva da força peso. São móbiles com essas características que participam dos torneios denominados gravity car race. Como os móbiles carentes de força de propulsão podem ser vistos como um caso particular (correspondente a $F=0$ ) do caso mais geral analisado na secção precedente (em que se considerou $F$ qualquer), é possível se servir do equacionamento analítico desenvolvido anteriormente, bastando para tanto fazer $F=0$ nas equações obtidas. Assim procedendo, é 
legítimo concluir que, na equação diferencial (2) que modela a velocidade dos móbiles, a velocidade limite, expressa pela equação (3), assume a forma: $v_{L}=\frac{\alpha}{k}=\frac{m \cdot \alpha}{K}$.

A partir da equação (4), pode-se concluir que a equação de velocidade será dada por:

$$
v(t)=v_{L}\left(1-e^{-k t}\right)=\frac{\alpha}{k}\left(1-e^{-k t}\right) .
$$

E a partir da equação (5), pode-se inferir que a equação de posição será dada por:

$$
s(t)=\frac{\alpha}{k}\left[t+\frac{1}{k}\left(e^{-k t}-1\right)\right] .
$$

Em um determinado intervalo de tempo $t$, previamente definido, estes móbiles percorrem uma distância s sobre a rampa expressa pela equação (24). Procurando analisar o efeito que possui a massa desses móbiles sobre seu desempenho, isto é, sobre a distância que conseguem percorrer nesse intervalo de tempo $t$ previamente definido, a equação de posição (24) pode ser reescrita na forma:

$$
s(k)=\frac{\alpha}{k^{2}}\left(k t+e^{-k t}-1\right) .
$$

Na equação (25), fazendo $k=K / m$, obtém-se:

$$
s(m)=\frac{\alpha m}{K^{2}}\left[K t+m\left(e^{-\frac{K}{m} t}-1\right)\right] .
$$

Visando analisar como varia o desempenho $s$ em função da massa $m$ dos móbiles cabe perguntar, em primeiro lugar, se a função de posição $s(m)$ possuiria um extremante. Ora, na secção anterior demonstrou-se que a função de posição somente admite um extremante quando a força de tração $F$ situa-se no intervalo: $(\sqrt{2}-1) . \alpha K t<F<\alpha K t$. Como $F=0$ não se encontra nesse intervalo, evidentemente, é forçoso concluir que a função $s(m)$ não admite um extremante. Com efeito, é possível verificar que a derivada $\frac{d s}{d m}$ é sempre positiva; consequentemente, a função $s(m)$ será sempre crescente. Isso significa que quanto maior for a massa dos veículos, maior será a distância $s$ percorrida no intervalo de tempo estipulado para a competição. No entanto, há um valor limite para a distância percorrida: por maior que seja a massa dos móbiles, esta distância limite jamais poderá ser superada. De fato, servindo-se do cálculo dos limites da função de posição obtidos na equação (14), em que se deve fazer $F=0$, e na equação (16), pode-se concluir que:

$$
\lim _{m \rightarrow 0^{+}} s(m)=0 \quad \text { e } \quad \lim _{m \rightarrow \infty} s(m)=\frac{\alpha t^{2}}{2} .
$$


O gráfico da Figura 9 representa o comportamento da distância percorrida $s$, no intervalo de tempo $t$ da competição, em função da massa $m$ dos móbiles. Observa-se que a distância cresce continuamente com a massa, partindo do zero e tendendo a um valor assintótico no infinito dado por $s_{L}=\alpha t^{2} / 2$. O valor da abscissa $m_{0}$ indicada no gráfico corresponde a $m_{0}=(10 / 3) K t$ e será explicado mais adiante.

Figura 9 - Gráfico da posição $s$ (ordenada) em função da massa $m$ (abscissa). Curva apresenta comportamento assintótico no infinito.

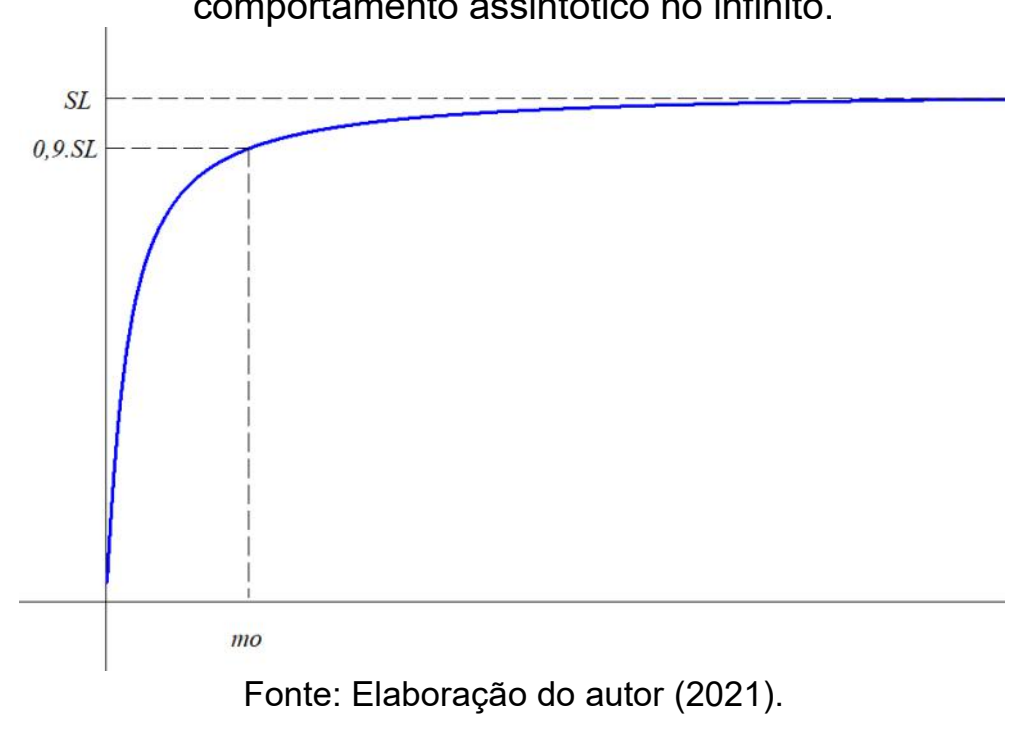

Como o aumento da massa dos veículos poderia ser realizado sem dificuldade pelos competidores indefinidamente, uma competição com veículos sem propulsão (sejam eles pilotados ou teleguiados) precisaria estipular um valor de massa para todos os veículos da competição. Uma pesagem criteriosa precisaria, então, ser realizada para garantir que a massa total dos móbiles (incluindo a massa do piloto, caso sejam pilotados) fosse rigorosamente a mesma. Nesse caso, o desafio a ser enfrentado por cada equipe de competidores consistiria em buscar minimizar, recorrendo aos mais variados artifícios e aprimoramentos técnicos, tanto a constante de arrasto aerodinâmico como o coeficiente de atrito de rolamento de seus móbiles, pois é uma redução desses parâmetros que poderia proporcionar um ganho de desempenho.

Para uma competição proposta nestes termos, a questão que caberia formular diz respeito aos critérios que deveriam ser observados para definir o valor de massa mais apropriado a ser estipulado para os veículos competidores.

Fazendo algumas ponderações nesse sentido, resultantes de uma análise do comportamento da função $s(m)$ expressa na equação (26), conviria observar que para $m=(10 / 3) K t$ a distância percorrida corresponderá a aproximadamente $90 \%$ do valor assintótico $s_{L}$. Na Figura 9, esse valor de massa está designado como $m_{0}$. A determinação da massa dos móbiles por esse critério poderia ser adotada caso fosse desejável que, no tempo estipulado para a competição, os veículos percorressem uma distância próxima da máxima possível, por maior 
que fosse a massa que tivessem. Com essa escolha, vale notar que pequena será a sensibilidade do desempenho a possíveis variações de massa, o que depõe a favor da adoção desse critério. No entanto, para este valor de massa, os veículos estarão longe de atingir o regime estacionário no tempo da competição. Isto porque, ao tomar $K t=0,3 m$ o critério para regime estacionário não se verifica, posto que esse critério exige $K t>3 m$. De fato, pode-se calcular que atingirão apenas $26 \%$ da velocidade limite. Em contrapartida, caso se desejasse que os veículos terminassem a competição no regime estacionário, seria preciso escolher para a massa dos veículos um valor, no mínimo, dez vezes menor, isto é escolher $m \leq(1 / 3) K t$. Assim fazendo, porém, a distância percorrida no tempo da competição será de, no máximo, $45 \%$ do valor assintótico $S_{L}$. Nessa situação, grande será a sensibilidade do desempenho a pequenas variações de massa, o que fica patente mediante uma simples análise do gráfico apresentado na Figura 9, que revela uma acentuada inclinação da curva para estes valores de massa. Por esse motivo, os veículos precisarão ser pesados de forma muito cuidadosa e criteriosa (considerando, inclusive, a massa conjunta de seus pilotos na pesagem, caso pilotados), para evitar que eventuais diferenças de massa entre os veículos, por menores que sejam, acabem por favorecer ou desfavorecer de forma velada alguns competidores.

\subsection{Sensibilidade do desempenho a variações de massa}

Pode-se empregar o cálculo de diferenciais para realizar uma estimativa da variação na posição $d s$ ocasionada por uma variação incremental $d m$ de massa. Para tanto, deve-se escrever que: $d s=s_{m} \cdot d m$, onde $s_{m}=\frac{d s}{d m}$. A derivada da função $s(m)$ em relação à massa, realizada a partir da equação (26), fornece: $\frac{d s}{d m}=\frac{\alpha}{m k^{2}}\left[(k t-2)+(k t+2) e^{-k t}\right]$. Desse modo:

i) Caso se tome $t=T_{e} \Rightarrow k t=3$ :

$$
\left.s_{m}\right|_{k t=3}=\frac{\alpha}{m k^{2}}\left(1+5 e^{-3}\right) \simeq 1,25 \frac{\alpha}{m k^{2}} \quad \text { e }\left.\quad s\right|_{k t=3}=\frac{\alpha}{k^{2}}\left(2+e^{-3}\right) \simeq 2,05 \frac{\alpha}{k^{2}}
$$

Por conseguinte, a variação percentual da posição provocada por uma variação incremental $d m$ de massa será, neste caso, dada por:

$$
\left.\frac{d s}{s}\right|_{k t=3} \times 100 \%=\left.\frac{s_{m} \cdot d m}{s}\right|_{k t=3} \times 100 \%=\frac{d m}{m} 61 \%
$$

ii) No entanto, caso se tome $k t=3 / 10$ :

$$
\left.s_{m}\right|_{k t=3 / 10}=\frac{\alpha}{m k^{2}}\left(23 e^{-3 / 10}-17\right) \times 10^{-1} \simeq 3,88 \times 10^{-3} \frac{\alpha}{m k^{2}}
$$

e 


$$
\left.S\right|_{k t=3 / 10}=\frac{\alpha}{k^{2}}\left(e^{-3 / 10}-7 / 10\right) \simeq 4,08 \times 10^{-2} \frac{\alpha}{k^{2}} .
$$

Consequentemente, a variação percentual da posição ocasionada por uma variação incremental $d m$ de massa será, neste caso:

$$
\left.\frac{d s}{s}\right|_{k t=3 / 10} \times 100 \%=\left.\frac{s_{m} \cdot d m}{s}\right|_{k t=3 / 10} \times 100 \%=\frac{d m}{m} 9,5 \%
$$

Os resultados expressos nas equações (27) e (28) atestam, como era esperado, uma sensibilidade do desempenho a eventuais variações de massa dos veículos muito acentuada no primeiro caso (quando $m=1 / 3 K t$ ) e bem mais baixa no segundo caso (quando $m=10 / 3 K t$ ). Para ser mais exato, a sensibilidade do desempenho se torna seis vezes e meia menor quando a massa passa a ser dez vezes maior.

\subsection{Roteiro para definição dos termos da competição}

Considerando que se disponha, para realização da competição, de uma rampa retilínea com inclinação constante $\theta$ em relação à horizontal e comprimento total $L$, o seguinte roteiro poderia ser sugerido para definição dos critérios da competição.

i) Em primeiro lugar, seria preciso que cada equipe consiga estimar experimentalmente, para seu veículo, o valor da constante de arrasto $K$ e coeficiente de atrito de rolamento $\mu$. Este é um trabalho de física experimental que deverá ser realizado previamente e que poderá, inclusive, se servir de alguns dos equacionamentos aqui desenvolvidos.

Feito isto, pode-se calcular o valor médio da constante de arrasto $\bar{K}$ de todos os veículos participantes, assim como o valor médio $\bar{\mu}$ dos coeficientes de atrito de rolamento - e com ele calcular o valor médio $\bar{\alpha}=g(\operatorname{sen} \theta-\bar{\mu} \cos \theta)$.

ii) Em seguida, pode-se determinar o tempo total $t$ da competição a partir da extensão $L$ da rampa disponível. Tem-se aqui duas possibilidades:

Caso se queira adotar o critério segundo o qual os veículos deverão percorrer, na extensão da rampa, $90 \%$ do valor assintótico $s_{L}=\alpha t^{2} / 2$, segue então que

$$
L=\frac{9}{10} S_{L} \Rightarrow \frac{9}{20} \bar{\alpha} t^{2}=L \Rightarrow t \simeq 1,49 \sqrt{L / \bar{\alpha}} \text {. }
$$

Neste caso, a massa total $m$ que os móbiles deverão possuir será determinada pela expressão $m=\frac{10}{3} \bar{K} t=4,97 \bar{K} \sqrt{L / \bar{\alpha}}$.

Caso, no entanto, se escolha adotar o critério segundo o qual os veículos deverão atingir o regime estacionário ao final da rampa, é preciso que 


$$
L=\frac{41}{90} s_{L} \Rightarrow \frac{41}{180} \bar{\alpha} t^{2}=L \Rightarrow t \simeq 2,10 \sqrt{L / \bar{\alpha}} .
$$

Então, a massa total $m$ dos veículos será dada por $m=\frac{1}{3} \bar{K} t=0,70 \bar{K} \sqrt{L / \bar{\alpha}}$.

Vale notar que a massa dos móbiles será, neste caso, cerca de sete vezes menor que aquela estipulada pelo critério anterior e que, como ressaltado anteriormente, grande será a sensibilidade do desempenho a pequenas variações de massa.

\subsection{Sensibilidade do desempenho a variações dos parâmetros}

Uma estimativa da variação da posição $d s$ provocada por variações incrementais $d K$ e $d \mu$ que incidem tanto sobre a constante de arrasto como sobre o coeficiente de atrito de rolamento, respectivamente, pode ser obtida por meio do cálculo da diferencial total $d s$ :

$$
d s=\frac{\partial s}{\partial K} d K+\frac{\partial s}{\partial \mu} d \mu
$$

Cada uma das derivadas parciais que aparecem na equação (29) pode ser determinada pelo emprego da regra da cadeia. A partir da equação (25), é possível calcular que: $\frac{\partial s}{\partial k}=-\frac{\alpha}{k^{3}}\left[(k t-2)+(k t+2) e^{-k t}\right]$. Portanto, a primeira derivada parcial resulta em:

$$
s_{K}=\frac{\partial s}{\partial K}=\frac{\partial s}{\partial k} \frac{d k}{d K}=\frac{1}{m} \frac{\partial s}{\partial k}=-\frac{\alpha}{m k^{3}}\left[(k t-2)+(k t+2) e^{-k t}\right]
$$

Para a segunda, como $\frac{\partial s}{\partial \alpha}=\frac{1}{k^{2}}\left(k t+e^{-k t}-1\right)$, temos que:

$$
s_{\mu}=\frac{\partial s}{\partial \mu}=\frac{\partial s}{\partial \alpha} \frac{d \alpha}{d \mu}=-g \cos \theta \cdot \frac{\partial s}{\partial \alpha}=-\frac{g \cos \theta}{k^{2}}\left(k t+e^{-k t}-1\right)
$$

Lembrando que, conforme a equação (25), $s=\frac{\alpha}{k^{2}}\left(k t+e^{-k t}-1\right)$, pode-se estimar a influência percentual $d s / s$ provocada pela variação de cada um dos parâmetros tomados separadamente.

i) Para pequenas alterações $d K$ nos valores da constante de arrasto $K$, a variação percentual relativa de desempenho $s$ será dada por:

$$
\begin{gathered}
\frac{s_{K}}{s}=-\frac{\left[(k t-2)+(k t+2) e^{-k t}\right]}{k m\left(k t+e^{-k t}-1\right)} \Rightarrow \\
\frac{s_{K} d K}{s} \times 100 \%=-\frac{\left[(k t-2)+(k t+2) e^{-k t}\right]}{\left(k t+e^{-k t}-1\right)} \frac{d K}{K} \times 100 \%
\end{gathered}
$$


ii) Para pequenas alterações $d \mu$ nos valores do coeficiente de atrito de rolamento $\mu$, a variação percentual relativa de desempenho s será dada por:

$$
\frac{s_{\mu}}{s}=-\frac{g \cos \theta}{\alpha}=-(\operatorname{tg} \theta-\mu)^{-1} \quad \Rightarrow \frac{s_{\mu} d \mu}{s} \times 100 \%=-\frac{d \mu}{\operatorname{tg} \theta-\mu} \times 100 \%
$$

iii) As expressões dadas pelas equações (30) e (31) podem ser úteis para determinar qual dos dois parâmetros seria mais vantajoso buscar reduzir por meio de aprimoramentos técnicos, uma vez que a redução de um deles pode, eventualmente, vir a proporcionar um ganho de desempenho muito maior que uma redução equivalente promovida no outro.

Com relação ao coeficiente de atrito de rolamento, vale notar, observando a equação (31), que o ganho de desempenho é inversamente proporcional à diferença $(\operatorname{tg} \theta-\mu)$, sendo independente do produto $k t$ e, portanto, da massa dos veículos. O mesmo não pode ser dito do ganho de desempenho relativamente à constante de arrasto $K$, a qual depende do produto $k t$, como evidencia a equação (30).

A título de comparação, caso o valor da massa $m$ escolhido seja tal que $k t=3$ (de modo que os veículos atinjam a velocidade limite ao final da rampa), tem-se:

$$
\left.\frac{s_{K} d K}{s}\right|_{k t=3} \times 100 \%=-\frac{d K}{K} 61 \%
$$

No entanto, caso se escolha para a massa $m$ dos veículos um valor dez vezes maior, fazendo $k t=3 / 10$ (de modo que os veículos atingirão apenas $26 \%$ da velocidade limite ao final da rampa), tem-se:

$$
\left.\frac{s_{K} \cdot d K}{s}\right|_{k t=3 / 10} \times 100 \%=-\frac{d K}{K} 9,5 \%
$$

No primeiro caso, um pequeno decréscimo $d K$ na constante de arrasto acarretará um aumento de aproximadamente $61(d K / K) \%$ na distância percorrida. No segundo caso, em que a massa é dez vezes maior, o mesmo decréscimo $d K$ acarretará, no entanto, um aumento 6,4 vezes menor, de apenas $9,5(d K / K) \%$ na distância percorrida.

\subsection{Algumas curvas ilustrativas}

As curvas de desempenho apresentadas a seguir foram traçadas empregando-se a função $s(m)$ dada pela equação (26), que expressa como varia o desempenho $s$ (distância percorrida no intervalo de tempo $t$ estipulado pela competição) em função da variação da massa $m$ dos móbiles. Em todos os gráficos, a distância $s$ (em metros) está disposta no eixo das ordenadas e a massa $m$ (em $\mathrm{kg})$ no eixo das abscissas. 
i) Para a curva de desempenho apresentada na Figura 10, foram utilizados os seguintes parâmetros: $K=1 \mathrm{~N} . \mathrm{s} / \mathrm{m} ; \alpha=1$ e $t=50$ segundos. Neste caso, temos: $s_{L}=\alpha t^{2} / 2=1225$ metros; $m_{o}=(10 / 3) K t=150 \mathrm{~kg}$.

Figura 10 - Gráfico da posição $s$ (ordenada) em função da massa $m$ (abscissa). Assíntota em $s_{L}=1225$ metros.

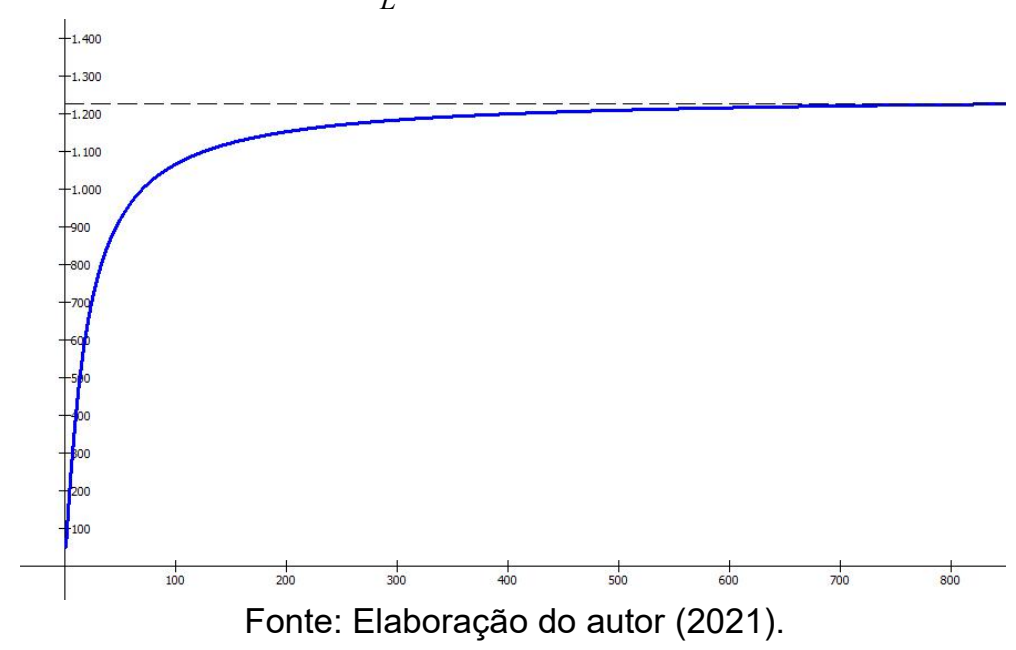

ii) Para as curvas de desempenho traçadas na Figura 11, foram mantidos constantes os parâmetros $K=1 \quad \mathrm{~N} . \mathrm{s} / \mathrm{m}$ e $t=50$ segundos, fazendo-se variar a constante $\alpha=g(\operatorname{sen} \theta-\mu \cos \theta)$. Evidentemente, isso significa variar o coeficiente de atrito de rolamento $\mu$, pois a inclinação $\theta$ é uma constante para cada competição. As curvas permitem inferir que a sensibilidade do desempenho relativamente às variações da constante $\mu$ (em termos da variação percentual relativa) não se deixa afetar pela massa. Esse comportamento é condizente com a equação (31), de acordo com a qual o ganho de desempenho relativamente a variações no coeficiente de atrito de rolamento independe da massa dos veículos.

Figura 11 - Gráfico da posição $s$ (ordenada) em função da massa $m$ (abscissa) para distintos valores da constante $\alpha$.

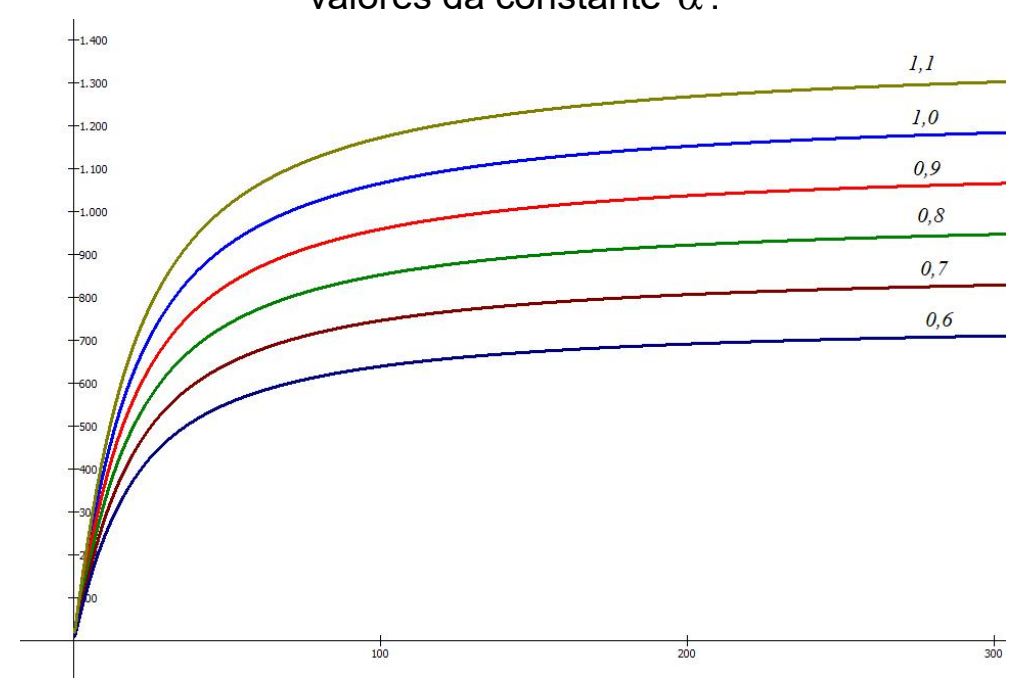

Fonte: Elaboração do autor (2021). 
iii) Para as curvas de desempenho traçadas na Figura 12, foram mantidos constantes os parâmetros $\alpha=1$ e $t=50$ segundos, fazendo-se variar a constante de arrasto $K$.

Figura 12 - Gráfico da posição $s$ (ordenada) em função da massa $m$ (abscissa) para distintos valores da constante de arrasto $K$.

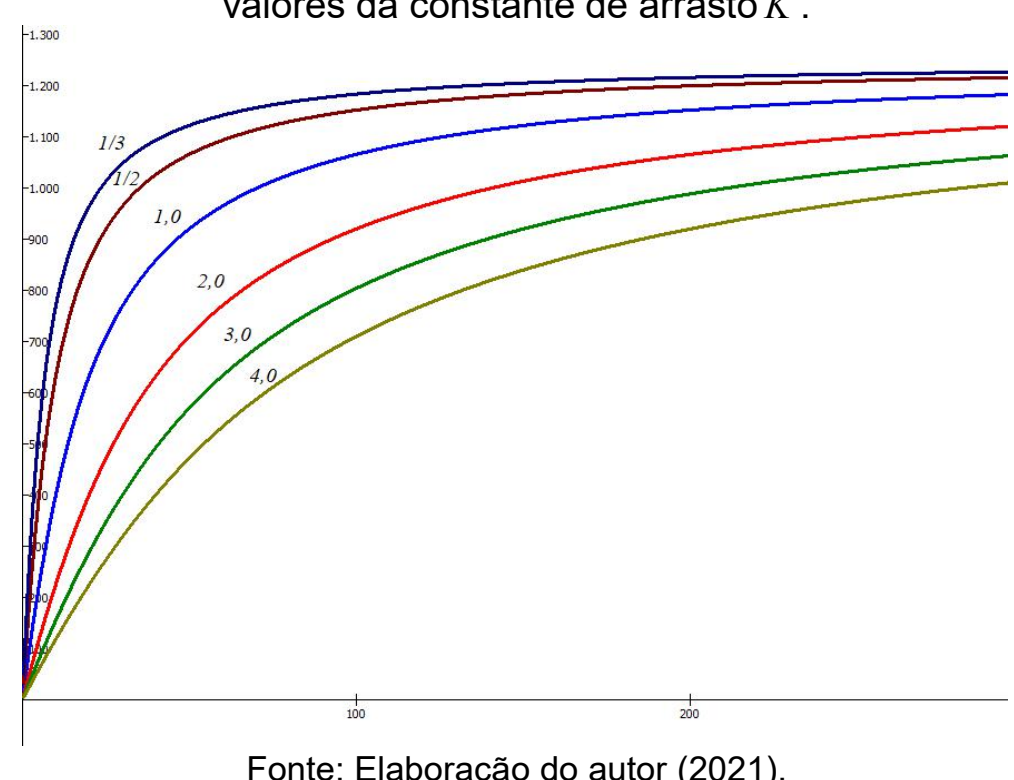

Fonte: Elaboração do autor (2021).

Como se pode observar, as curvas da Figura 12 revelam sensibilidade menor do desempenho às variações da constante $K$ para valores maiores de massa: com o aumento da massa, a sensibilidade tende a ser menor.

\section{Considerações finais}

A analítica de móbiles em descida de rampa aqui apresentada procurou fornecer os subsídios da análise matemática para uma eventual competição em que veículos, pilotados ou teleguiados, disputam o desafio de percorrer a maior distância sobre uma rampa em certo intervalo de tempo previamente estipulado. Duas modalidades de competição foram analisadas.

Em um primeiro momento, realizou-se a análise da modalidade em que os veículos são dotados de propulsão própria, geradora de uma força de tração constante. A análise permitiu concluir que, para essa modalidade de competição, existe um valor ótimo de massa que maximiza o desempenho, desde que a força de tração se encontre dentro de uma faixa de variação determinada. Em virtude desse comportamento, pareceu interessante propor um critério que exigisse das equipes competidoras, ao buscar melhorar o desempenho de seus veículos, não apenas minimizar as forças de arrasto aerodinâmico e de atrito de rolamento graças a aprimoramentos técnicos, mas também procurar otimizar a massa de seus veículos. $A$ analítica desenvolvida nesse trabalho obteve uma expressão para cálculo do valor ótimo de massa em função dos parâmetros estipulados para a competição, bem como das constantes associadas ao arrasto aerodinâmico e ao atrito de rolamento dos veículos, que precisariam ser determinadas experimentalmente. Essa expressão pode ser útil às equipes competidoras desde que, no 
intervalo de tempo estipulado para a competição, os veículos atinjam uma velocidade próxima do valor da velocidade limite. Por essa razão, o critério sugerido para essa modalidade propôs a adoção de um tempo de competição que seja igual ou maior ao tempo necessário para ingresso no regime estacionário. Em competições nas quais esse critério não se verificar, a expressão obtida analiticamente neste estudo não poderá ser empregada. Nesse caso, a determinação do ponto de máximo desempenho deverá ser realizada por meio de métodos numéricos, uma vez que a equação a ser resolvida é uma equação transcendente.

Em um segundo momento, considerou-se o caso em que os móbiles não são dotados de força de propulsão própria e descem a rampa exclusivamente pela ação da gravidade. Nessa modalidade, conhecida por gravity car race, verificou-se que o desempenho cresce continuamente com a massa. Por esse motivo, recomendou-se que, para uma competição nessa modalidade, fosse estipulado um valor de massa único para todos os veículos competidores. Esse critério foi ainda refinado, propondo a adoção de um valor padrão capaz de propiciar uma baixa sensibilidade do desempenho a eventuais incorreções da massa real dos móbiles, as quais poderiam passar despercebidas. Sendo a massa idêntica para todos os móbiles, o ganho de desempenho seria então obtido graças aos mais variados aprimoramentos técnicos, empreendidos pelas equipes competidoras, capazes de proporcionar uma redução significativa quer na constante de arrasto aerodinâmico, quer no coeficiente de atrito de rolamento de seus veículos.

\section{Referências}

BRUNETTI, F. Mecânica dos Fluidos. São Paulo: Pearson, 2008.

COLETTA, V. P.; EVANS, J. Analysis of a model race car. American Journal of Physics, v. 76, $n$. 10, p. 903-907, 15 set. 2008. DOI: https://doi.org/10.1119/1.2955793.

COWLEY, E. R. Pinewood Derby Physics. The Physics Teacher, v. 27, p. 610-612, nov. 1989. DOI: https://doi.org/10.1119/1.2342889.

DRISCOLL, H.; BULLAS, A.; KING, C.; SENIOR, T.; HAAKE, S.; HART, J. Application of Newtonian Physics to predict the speed of a gravity racer. Physics Education, v. 51, n. 4, p. 1-7, 4 maio 2016. DOI: https://doi.org/10.1088/0031-9120/51/4/045002.

HALLIDAY, D.; RESNICK, R. Fundamentos de Física, v. 1, Rio de Janeiro: Gen-LTC, 2016.

JOBE, J. D. The physics of the pinewood derby with engineering applications. Missouri: Publicação do Próprio Autor, 2004.

MANN, B. P; GIBBS, M. M.; SAH, S. M. Dynamics of a gravity car race with application to the Pinewood Derby. Mechanical Sciences, v. 3, p. 73-84, 4 dez. 2012. DOI:

https://doi.org/10.5194/ms-3-73-2012.

MARTIN, J. K. Factors Influencing the Forward Acceleration of a Gravity Powered Soapbox Race Vehicle. British Journal of Applied Science \& Technology, v. 12, n. 6, p. 1-9, 3 nov. 2015. DOI: https://doi.org/10.9734/bjast/2016/17077. 
MEADE, D. Pinewood derby speed secrets. Londres: Dorling Kindersley Publishing, 2006.

NAGLE, R. K.; SAFF, E. B.; SNIDER, A. D. Equações Diferenciais. São Paulo: Pearson, 2012.

PEDIGO, T. How to Win a Pinewood Derby. Salt Lake: Winning Edge Publications, 2002.

POLYCARPOU, A.; SOLZAK, T. Engineering Outreach to Cub Scouts with Hands-on Activities Pertaining to the Pinewood Derby Car Race. International Journal of Engineering Education, v. 22, n. 5, p.1077-1096, 2006. Disponível em: https://www.ijee.ie/articles/Vol22-5/20 ijee1789.pdf. Acesso em: 3 ago. 2021.

RADE, D. Cinemática e Dinâmica para Engenharia. São Paulo: Gen-LTC, 2017.

THOMAS, G. B.; WEIR, M. D.; HASS, J. Cálculo, v. 1, São Paulo: Pearson Education, 2011.

TORVI, D. Using Pine Wood Derby Cars to Introduce Mechanical Engineering to Students in a First Year General Engineering Design Course. Proceedings of the Canadian Engineering Education Association (CEEA), Proceedings of CDEN/C2E2 Conference: University of Manitoba Winnipeg, Manitoba, July 22-24, 2007. 9 ago. 2011. DOI:

https://doi.org/10.24908/pceea.v0i0.3805.

ZILL, D. G.; CULLEN, M. R. Equações Diferenciais. v. 1. São Paulo: Pearson Education, 2007. 